九州大学学術情報リポジトリ

Kyushu University Institutional Repository

\title{
Almost limiting short-crested gravity waves in deep water
}

Okamura, Makoto

Research Institute for Applied Mechanics, Kyushu University

http://hdl. handle. net/2324/1909891

出版情報: Journal of Fluid Mechanics. 646, pp.481-508, 2010-02-10. Cambridge University Press (CUP)

バージョン :

権利関係 : ๑ Cambridge University Press 2010 


\title{
Almost-limiting short-crested gravity waves in deep water
}

\author{
MAKOTO OKA MURA \\ Research Institute for Applied Mechanics, Kyushu University, Kasuga 816-8580, Japan
}

(Received 30 April 2009 and in revised form 4 October 2009)

We investigate the properties of almost-limiting short-crested gravity waves with harmonic resonance for various incident angles. When the incident angle is less than $47.5^{\circ}$, the enclosed crest angle in non-resonant limiting waves is $90^{\circ}$, which corresponds to that in standing waves. In contrast, when the incident angle exceeds $47.5^{\circ}$, the enclosed crest angle in non-resonant limiting waves is $120^{\circ}$, which corresponds to that in twodimensional progressive waves. The enclosed crest angle is $90^{\circ}$ in resonant limiting waves for all incident angles. The crest becomes flatter than the trough in resonant limiting waves if the fundamental mode has a different sign from its harmonic resonant mode. Bifurcation of short-crested waves is also investigated.

\section{Introduction}

Three-dimensionality is important in nature because three-dimensional water waves occur more frequently in nature than two-dimensional ones. Weakly nonlinear threedimensional water waves in shallow water have been actively investigated for a few decades (Akylas (1994)). In particular, long waves propagating predominantly in one direction have been studied in detail because their time-evolution equation, called the Kadomtsev-Petviashvili (KP) equation, has been derived. The KP equation extends the Korteweg-de Vries equation by incorporating weakly three-dimensional effects. However, there have been few studies that go beyond weakly nonlinear theory in shallow water (Tanaka (1993)). In deep water, even weakly nonlinear three-dimensional water waves have not been investigated to the same degree because the two-dimensional coupled nonlinear Schrödinger equation, which corresponds to the KP equation, has a serious defect of energy leakage, which means that the energy initially confined to the uniform wave train and a pair of unstable perturbation modes eventually spreads to arbitrarily high modes (Martin \& Yuen (1980)). Many studies on short-crested waves have investigated wave profiles having moderate rather than large amplitudes (Hsu et al. (1979); Kimmoun et al. (1999); Ioualalen et al. (2006)) and their stability (Ioualalen \& Kharif (1993); Ioualalen \& Okamura (2002); Dias \& Kharif (1999)). Therefore, with the exception of the study on highly nonlinear short-crested waves in deep water by Roberts (1983), there is insufficient information on highly nonlinear three-dimensional water waves for both shallow and deep water.

The purpose of the present paper is to clarify the characteristics of limiting shortcrested waves in deep water. Linearly, a short-crested wave is defined as superposition of an incident two-dimensional progressive wave with an incident angle $\theta$ and its reflected wave. Hence, a short-crested wave tends to a two-dimensional progressive wave and a twodimensional standing wave as $\theta \rightarrow 90^{\circ}$ and $\theta \rightarrow 0^{\circ}$, respectively. Since the pioneering work by Yamada (1957), two-dimensional limiting progressive waves have been investigated 
in detail (Okamoto \& Shoji (2001)). The enclosed crest angle of the limiting wave is $120^{\circ}$ with a maximum wave steepness of 0.44316 (Williams (1981)). Few studies have been conducted on limiting standing waves (Dias \& Bridges (2006)). Of these studies, three have estimated the maximum wave steepness of limiting standing waves: 0.6202 (Mercer \& Roberts (1992)), 0.641 (Tsai \& Jeng (1994)) and 0.6272 (Okamura (2003)). These three values vary by about $3 \%$, whereas the maximum wave steepness of twodimensional progressive waves has been estimated with an error of less than $0.01 \%$. The limiting wave profile of the standing wave has a sharp crest, and it has been shown to have an enclosed crest angle of $90^{\circ}$ analytically by Penney \& Price (1952) and Okamura (1998) and numerically by Tsai \& Jeng (1994) and Okamura (2003). In two-dimensional progressive waves, the sharpness at the crest disappears after a transformation, whereas there is no useful transformation that removes the singular point at the sharp crest of standing waves. Thus, it is difficult to numerically obtain limiting standing waves.

There are two difficulties in obtaining the profile of a short-crested wave. One difficulty is caused by the radius of convergence being much smaller than the maximum wave steepness. The other difficulty is associated with the division by zero due to harmonic resonance. Harmonic resonance occurs if the linear wave frequency $\Omega\left(k_{x}, k_{y}\right)$ of the shortcrested wave satisfies $\Omega\left(j k_{x}, l k_{y}\right)=j \Omega\left(k_{x}, k_{y}\right)$, where $\Omega\left(k_{x}, k_{y}\right)=g\left(k_{x}^{2}+k_{y}^{2}\right)^{1 / 4}$ is the linear dispersion relation. The Padé approximant method overcomes the former difficulty and is useful for obtaining finite-amplitude non-resonant short-crested waves, with the exception of very-large-amplitude waves. However, it is unable to overcome the second difficulty (Roberts (1983)). Finite-amplitude resonant short-crested waves can be calculated by another method that includes the collocation method (Ioualalen et al. (2006); Okamura (1996)), which was proposed by Tsai \& Jeng (1994) for standing waves. This method resolves the two above-mentioned difficulties but it is not useful for obtaining very-large-amplitude waves. Here, the Galerkin method proposed by Okamura (2003) for standing waves is used instead of the collocation method to calculate almost-limiting short-crested waves.

The present paper is organised as follows. In $\S 2$, we present the formation of the problem. In $\S 3$, a numerical method is introduced for computing short-crested waves and two-dimensional progressive waves. In $\S 4$, we evaluate the reliability of the solutions by examining their convergence and by investigating the enclosed crest angles of limiting waves for the standing wave and the two-dimensional progressive wave. In $\S 5$, we present the maximum wave steepness, bifurcation diagrams and almost-limiting wave profiles for various incident angles. Section 6 summarises the findings.

\section{Formulation of the problem}

We consider the three-dimensional irrotational flow of an inviscid and incompressible fluid to treat one of the simplest three-dimensional progressive water waves, the shortcrested wave. Surface tension effects are omitted, although they become important near a sharp crest even for small capillarity (Dias \& Kharif (1999), Perlin \& Schultz (2000)). To express all the equations in non-dimensional form, we introduce the following dimensionless quantities

$$
\left(x^{*}, y^{*}, z^{*}, H^{*}\right)=K(x, y, z, \eta), \quad t^{*}=\omega t, \quad \Phi^{*}=\frac{K^{2}}{\omega} \phi, \quad G=\frac{K}{\omega^{2}} g,
$$

where $x$ and $y$ are the horizontal coordinates, $z$ is the vertical coordinate, $\eta(x, y, t)$ is the surface elevation, $K$ is the wavenumber of the incident wave, $\omega$ is the wave frequency of the short-crested wave to be treated, $\phi(x, y, z, t)$ is the velocity potential and $g$ is 
the gravitational acceleration. Note that the dimensionless quantity $G$ depends on the unknown $\omega$. Because we assume that the short-crested wave propagates in the $x$-direction without any change in its form, it is useful to introduce a moving coordinate system

$$
T=p x^{*}-t^{*}, \quad Y=q y^{*}, \quad Z=z^{*},
$$

where $p=\sin \theta$ and $q=\cos \theta$. Note the relations

$$
k_{x}=K \sin \theta, \quad k_{y}=K \cos \theta, \quad \tan \theta=k_{x} / k_{y},
$$

where $\left(k_{x}, k_{y}\right)$ is a wavenumber vector of the short-crested wave. Thus, we can treat the short-crested wave with a period of $2 \pi$ in $T$ and $Y$. By introducing

$$
\Phi(Y, Z, T)=\Phi^{*}\left(x^{*}, y^{*}, z^{*}, t^{*}\right), \quad H(Y, T)=H^{*}\left(x^{*}, y^{*}, t^{*}\right),
$$

we obtain non-dimensional governing equations in the moving coordinate system as follows. Laplace's equation:

$$
p^{2} \Phi_{T T}+q^{2} \Phi_{Y Y}+\Phi_{Z Z}=0 \quad \text { for } \quad Z<H(Y, T),
$$

the dynamic boundary condition:

$$
P(Y, Z, T)=-\Phi_{T}+\frac{1}{2}\left(p^{2} \Phi_{T}^{2}+q^{2} \Phi_{Y}^{2}+\Phi_{Z}^{2}\right)+G Z=0 \quad \text { on } \quad Z=H(Y, T),
$$

the kinematic boundary condition:

$$
\begin{aligned}
Q(Y, Z, T)= & \Phi_{T T}+p^{2} \Phi_{T}\left(-2 \Phi_{T T}+p^{2} \Phi_{T} \Phi_{T T}+q^{2} \Phi_{Y} \Phi_{Y T}+\Phi_{Z} \Phi_{Z T}\right) \\
& +q^{2} \Phi_{Y}\left(-2 \Phi_{Y T}+p^{2} \Phi_{T} \Phi_{Y T}+q^{2} \Phi_{Y} \Phi_{Y Y}+\Phi_{Z} \Phi_{Y Z}\right)+\Phi_{Z}\left(-2 \Phi_{Z T}\right. \\
& \left.+p^{2} \Phi_{T} \Phi_{Z T}+q^{2} \Phi_{Y} \Phi_{Y Z}+\Phi_{Z} \Phi_{Z Z}+G\right)=0 \quad \text { on } \quad Z=H(Y, T),
\end{aligned}
$$

and the bottom boundary condition:

$$
\Phi_{Z} \rightarrow 0 \quad \text { as } Z \rightarrow-\infty
$$

where $Z=H(Y, T)$ gives the surface shape of the short-crested wave. Condition (2.4) corresponds to $D P / D t=0$, which expresses that the rate of change of the pressure following a particle on the free surface must vanish. This condition is much more complicated than the usual one. It is, however, very useful for obtaining the limiting short-crested wave with a sharp crest numerically because it does not contain any space derivatives of the free surface such as $H_{Y}$ in (2.4) (Okamura (2003); Tsai \& Jeng (1994)). We impose the velocity potential on two periodic conditions:

$$
\Phi(Y, Z, T)=\Phi(Y+2 \pi, Z, T), \quad \Phi(Y, Z, T)=\Phi(Y, Z, T+2 \pi)
$$

and three symmetric wave conditions:

$$
\begin{gathered}
\Phi(Y, Z, T)=\Phi(-Y, Z, T), \quad \Phi(Y, Z, T)=-\Phi(Y, Z,-T), \\
\Phi(Y, Z, T)=-\Phi(\pi-Y, Z, \pi-T) .
\end{gathered}
$$

Now, we introduce the wave steepness

$$
\epsilon=\frac{1}{2}[H(0,0)-H(\pi, 0)],
$$

which is half the peak-to-trough height for non-resonant waves at $T=0$. In the case of non-resonant waves, $H(0,0)$ and $H(\pi, 0)$ correspond to the maximum and minimum heights, respectively; this is not necessarily true for resonant waves. For example, consider $H(Y, 0)=\cos Y+B_{2} \cos 2 Y$ for $-\pi \leqslant Y \leqslant \pi$, and let $H(Y, 0)$ have the maximum value 

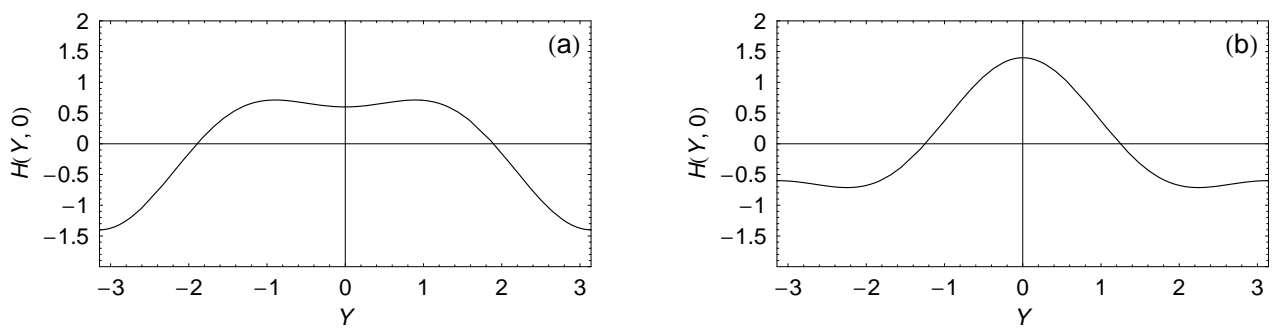

FiguRE 1. Surface shapes $H(Y, 0)=\cos Y+B_{2} \cos 2 Y$ at $T=0$ in the case of resonant waves: (a) $B_{2}=-0.4<0, Y_{\max }=\arccos (1 / 1.6) \approx 0.896, Y_{\min }=\pi$; (b) $B_{2}=0.4>0, Y_{\max }=0$, $Y_{\text {min }}=\arccos (-1 / 1.6) \approx 2.246$.

$H_{\max }=\max [H(Y, 0)]$ at $Y= \pm Y_{\max }$ and the minimum value $H_{\min }=\min [H(Y, 0)]$ at $Y= \pm Y_{\min }$. In the case $\left|B_{2}\right| \leqslant 1 / 4$, which corresponds to non-resonant waves $\left(\left|B_{2}\right| \ll 1\right)$, $Y_{\max }=0$ and $Y_{\min }=\pi$. In the case $B_{2}<-1 / 4, Y_{\max }=\arccos \left[-1 /\left(4 B_{2}\right)\right]$ and $Y_{\min }=\pi$, and in the case $B_{2}>1 / 4, Y_{\max }=0$ and $Y_{\min }=\arccos \left[-1 /\left(4 B_{2}\right)\right]$, both cases correspond to resonant waves $\left(B_{2}=O(1)\right)$, shown in figure 1 . Therefore, it is useful to introduce the effective wave steepness

$$
\epsilon^{*}=\frac{1}{2}\left(H_{\max }-H_{\min }\right),
$$

which represents half the difference of the maximum height $H_{\max }$ and the minimum height $H_{\min }$ at $T=0$.

The wave steepness defined in (2.9) is an overestimate for short-crested waves because the surface elevation $H$ is normalised by the incident wavenumber $K$ as in (2.1). Instead, we should use the wavenumber (or wavelength) of a short-crested wave for normalisation when estimating the wave steepness. Hence, it is reasonable to use the actual wave steepness $\tilde{\epsilon}$ defined as

$$
\tilde{\epsilon}= \begin{cases}\pi\left[\eta(0,0,0)-\eta\left(0, \lambda_{y}, 0\right)\right] / \lambda_{y} & \text { for } \quad 0^{\circ} \leqslant \theta \leqslant 45^{\circ} \\ \pi\left[\eta(0,0,0)-\eta\left(\lambda_{x}, 0,0\right)\right] / \lambda_{x} & \text { for } \quad 45^{\circ} \leqslant \theta \leqslant 90^{\circ}\end{cases}
$$

where $\lambda_{x}$ and $\lambda_{y}$ denote the $x$ - and $y$-direction wavelengths of the short-crested wave, respectively. Using $H(\pi, 0)=H(0, \pi)$ obtained from (2.8) and $2 \pi / K=\lambda_{x} \sin \theta=\lambda_{y} \cos \theta$, we can express the actual wave steepness as

$$
\tilde{\epsilon}=\epsilon \max (\cos \theta, \sin \theta),
$$

where $\max (a, b)$ denotes the maximum of $a$ and $b$.

Now, we give two limiting conditions for short-crested waves. The limiting condition of the standing wave corresponds to

$$
A_{c}^{*}=-\frac{1}{g} \frac{D \phi_{z}}{D t}=1 \quad \text { at } \quad z=\eta(0,0,0),
$$

which implies that the acceleration of a fluid particle at the crest (called the downward crest fluid acceleration) is equal to the gravitational acceleration $g$ at $t=0$ for the limiting wave. The limiting condition (2.11) is derived from the exact relation (Bridges (2009))

$$
\frac{D \phi_{x}}{D t}+\left(g+\frac{D \phi_{z}}{D t}\right) \eta_{x}=0
$$

because $D \phi_{x} / D t=0$ and $\eta_{x} \neq 0$ at the crest for the limiting wave. The limiting condition 
of the two-dimensional progressive wave corresponds to

$$
V_{c}^{*}=\frac{\phi_{x}}{c}=1 \quad \text { at } \quad z=\eta\left(c t_{0}, 0, t_{0}\right)
$$

which means that the fluid particle velocity at the crest, $x_{0}=c t_{0}$ (called the crest velocity) agrees with the phase velocity $c$ for the limiting progressive wave. The limiting condition (2.13) is derived from the usual kinematic boundary condition:

$$
\left(\phi_{X}-c\right) \eta_{X}=\phi_{z}, \quad X \equiv x-c t
$$

for progressive waves because $\phi_{z}=0$ and $\eta_{X} \neq 0$ at the crest for the limiting wave. It is important to note that $A_{c}^{*}=0$ if $V_{c}^{*}=1$, while $V_{c}^{*}=0$ if $A_{c}^{*}=1\left(\Phi_{Z T}=-G\right)$ because $A_{c}^{*}=-\Phi_{Z T}\left(1-p^{2} \Phi_{T}\right) / G$ and $V_{c}^{*}=p^{2} \Phi_{T}$ at the crest. In the case of short-crested waves, fluid acceleration is not necessary to reach the maximum at the crest and its direction is not necessarily vertically downward. Hence, (2.11) is not suitable, and (2.13) may also be inappropriate. Therefore, generalising (2.11) and (2.13), we introduce the following two limiting conditions for short-crested waves

$$
A_{c}=\frac{1}{g}\left|\frac{D}{D t} \nabla \phi\right|=\frac{1}{g} \sqrt{\left(\frac{D \phi_{x}}{D t}\right)^{2}+\left(\frac{D \phi_{y}}{D t}\right)^{2}+\left(\frac{D \phi_{z}}{D t}\right)^{2}}=1 \quad \text { at } \quad z=\eta\left(x_{0}, y_{0}, t_{0}\right)
$$

which means that the maximum acceleration of a fluid particle on the surface (called the surface fluid acceleration) is equal to the gravitational acceleration $g$ for the limiting wave at $t=0$, and

$$
V_{c}=\frac{\phi_{x}}{c}=1 \quad \text { at } \quad z=\eta\left(x_{0}, y_{0}, t_{0}\right),
$$

which implies that the maximum fluid particle velocity in the $x$-direction on the free surface (called the surface velocity) is equal to the phase velocity $c=\omega /(p K)$ for the limiting wave.

\section{Numerical method for computing short-crested waves}

We can express the velocity potential as a solution of Laplace's equation (2.2) with a truncated series under (2.5)-(2.8) as follows:

$$
\begin{gathered}
\Phi(Y, Z, T)=\sum_{k=0}^{N} \sum_{j=1}^{N} A_{k j} \cos (k Y) \exp \left(\alpha_{k j} Z\right) \sin (j T), \quad \alpha_{k j}=\sqrt{k^{2} q^{2}+j^{2} p^{2}}, \\
\boldsymbol{A}=\left\{A_{k j}\right\}=\left\{A_{11}, A_{02}, A_{22}, A_{13}, A_{31}, A_{33}, \ldots, A_{N N}\right\},
\end{gathered}
$$

where unknown coefficients $A_{k j}$ are non-zero due to (2.8) only if $k+j$ is even, and $N$ is the truncation number. The number of unknowns $\left(A_{k j}\right.$ and $\left.G\right)$ is $N(N+1) / 2+1$. We use Galerkin's method to obtain the same number of independent relations as unknowns. Substituting (3.1) into (2.3), we numerically obtain the free surface profile

$$
Z=H(Y, T)=H(Y, T ; \boldsymbol{A}, G)
$$

by Newton's method. Note that $H(Y, T)$ and $\Phi(Y, Z, T)$ also depend on the unknowns $\boldsymbol{A}$ and $G$. When $\theta=90^{\circ}$, the velocity potential and the surface profile are expressed by

$$
\Phi(Z, T)=\sum_{j=1}^{N-1} A_{j} \exp (j Z) \sin (j T), \quad Z=H(T)
$$


instead of by (3.1) and (3.2). Substituting (3.2) into (2.4), we obtain the independent relations:

$$
F_{l m}(\boldsymbol{A}, G)=\int_{0}^{\pi} d Y \int_{0}^{\pi} d T Q(Y, H(Y, T ; \boldsymbol{A}, G), T ; \boldsymbol{A}, G) \cos (l Y) \sin (m T)=0,
$$

which are evaluated with an $M$-point Fourier transform. The number of independent relations (3.4) is $M(M+1) / 2$ because (3.4) is trivial due to (2.8) if $l+m$ is odd. We obtain the same number of independent relations as unknowns $A_{k j}$ if $M=N$. The $N$-point is, however, too small to evaluate the integral (3.4) accurately in obtaining almost-limiting waves, and hence we set $M>N$. Therefore, we can obtain $N(N+1) / 2$ independent relations (3.4) for $0 \leqslant l \leqslant N$ and $1 \leqslant m \leqslant N$, satisfying $l+m$ is odd. Another independent relation is expressed by

$$
W(\boldsymbol{A}, G)=2 \epsilon-H(0,0 ; \boldsymbol{A}, G)+H(\pi, 0 ; \boldsymbol{A}, G)=0
$$

which is related to the wave steepness (2.9). Instead, we use the fixed fundamental mode condition

$$
W(\boldsymbol{A}, G)=\epsilon-A_{11}=0,
$$

when calculating bifurcation branches for resonant waves, and the crest velocity condition

$$
W(\boldsymbol{A}, G)=\epsilon-V_{c}^{*}=\epsilon-p^{2} \Phi_{T}=0,
$$

is useful when $\theta=85^{\circ}$ and $90^{\circ}$.

Finally, we obtain a sufficient number of independent relations and we can solve the nonlinear equations (3.4) and (3.5) for $A_{k j}$ and $G$ by Newton's method for various values of $\epsilon$ and $\theta$ if an initial solution of iteration is given for $\epsilon_{0}$ and $\theta_{0}$. The $\theta$-fixed condition, $\theta=\theta_{0}$, is used in almost all cases but the $\epsilon$-fixed condition, $\epsilon=\epsilon_{0}$, is used for calculating a distinct branch such as a loop, shown in figure $6(\mathrm{~b})$. Here the third-order short-crested wave obtained by $\mathrm{Hsu}$ et al. (1979) is used as the initial solution of iteration. We stop the iteration if the maximum difference between the unknowns before an iteration and that after the iteration is smaller than $10^{-9}$. Calculation of the Jacobian matrix, which is necessary for Newton's method, is shown in Appendix A.

\section{Convergence of solutions}

We examine the convergence of solutions by considering the dependence of coefficients $A_{k j}$ on $k$ and $j$ for almost-limiting waves in the cases $\theta=0^{\circ}, 25^{\circ}, 40^{\circ}, 70^{\circ}$ and $90^{\circ}$. Figure 2 shows $\left|A_{k j}\right|$ as a function of the maximum of $k$ and $j$ for six cases. In all these cases except for (d) $\left|A_{k j}\right|$ is less than $10^{-7}$ for $\max (k, j)=N$. In the case of (d), $\left|A_{k j}\right| \lesssim$ $10^{-4}$ for $\max (k, j)=N$, but because $\left|A_{k j}\right|$ decreases rapidly for $22 \leqslant \max (k, j) \leqslant N$ as $\max (k, j)$ increases it is expected that $\left|A_{k j}\right|<10^{-7}$ for larger values of $N$. Even for the worst case of $\theta=70^{\circ}$ the convergence becomes much better for the wave steepness of $\epsilon \approx 0.84 \epsilon_{\max }$ (a little smaller than the maximum wave steepness) in the case of (e) than for the maximum wave steepness $\epsilon_{\max }$ in the case of (d). Therefore, the convergence is reasonably satisfactory.

Table 1 lists the maximum wave steepness $\epsilon_{\max }$ and the corresponding surface fluid acceleration $A_{c}$ for various values of $N$ and $M$ in the case $\theta=25^{\circ}$. It is possible to calculate the short-crested waves up to $A_{c}=0.99 \ldots$ in the cases $N=20,25$ and up to $A_{c}=0.90 \ldots$ in the case $N=30$. We treat a square matrix of size $N(N+1) / 2+2$ in Newton's method; the truncation number $N$ of 30 is probably too large to accurately calculate the matrix to double precision due to rounding errors. Hence, $N=25$ and 

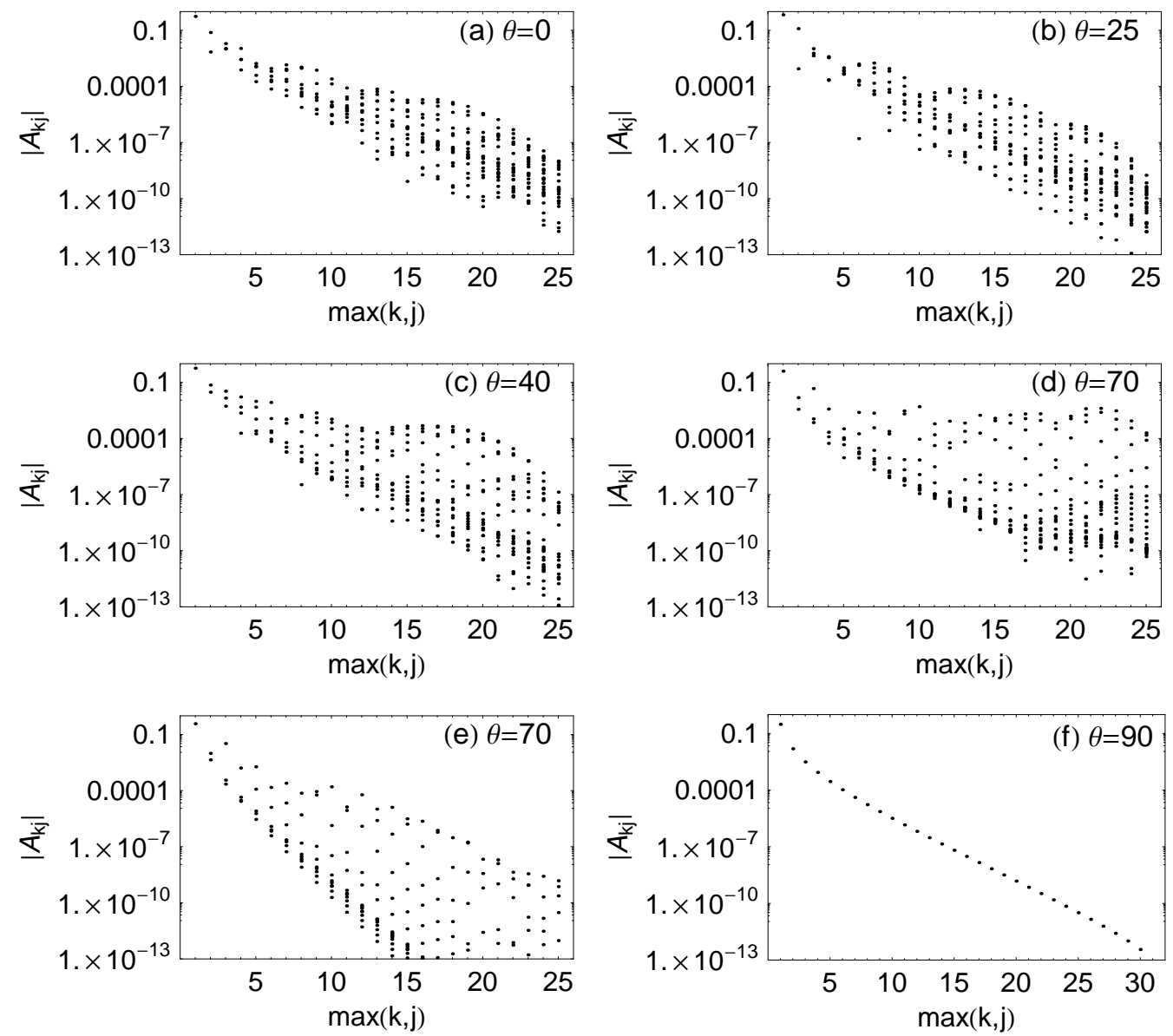

FiguRE 2. The absolute value of the coefficient $A_{k j}$ as a function of $\max (k, j)$ : (a) $\theta=0^{\circ}$, $A_{c}=0.9986$; (b) $\theta=25^{\circ}, A_{c}=0.9942$; (c) $\theta=40^{\circ}, A_{c}=0.9781$; (d) $\theta=70^{\circ}, V_{c}=0.9871$, $\epsilon=0.4788=\epsilon_{\max } ;(\mathrm{e}) \theta=70^{\circ}, V_{c}=0.5369, \epsilon=0.4 \approx 0.84 \epsilon_{\max } ;(\mathrm{f}) \theta=90^{\circ}, V_{c}=0.9630$, $\epsilon=0.4414$. $N=25, M=2^{8}$ for (a)-(e) and $N=32$ for (f).

$\begin{array}{rcccc}N & M & 2^{7} & 2^{8} & 2^{9} \\ 20 & (0.7954,0.9507) & (0.8069,0.9990) & (0.8073,0.9992) & (0.8073,0.9991) \\ 25 & (0.7981,0.9071) & (0.8052,0.9935) & (0.8060,0.9942) & (0.8061,0.9938) \\ 30 & (0.7670,0.8529) & (0.7910,0.9013) & (0.7910,0.9021) & (0.7911,0.9024)\end{array}$

TABLE 1 . The maximum wave steepness $\epsilon_{\max }$ and the corresponding surface fluid acceleration $A_{c}$, denoted by $\left(\epsilon_{\max }, A_{c}\right)$, for various values of $N$ and $M$ in the case $\theta=25^{\circ}$.

$M=2^{8}$ are used in all cases except for $\theta=90^{\circ}$. This table also indicates that the most significant figure is three for calculating the maximum wave steepness.

Figure 3 shows the almost-limiting wave profile near the crest in the case of a standing wave $\left(\theta=0^{\circ}\right)$ for $A_{c}=0.9986, N=25$ and $M=2^{8}$. This figure suggests that the enclosed crest angle of the limiting wave is $90^{\circ}$, which is the well-known enclosed crest angle of the standing wave (Penney \& Price (1952)). The velocity potential $\Phi$ is expanded in a Fourier 


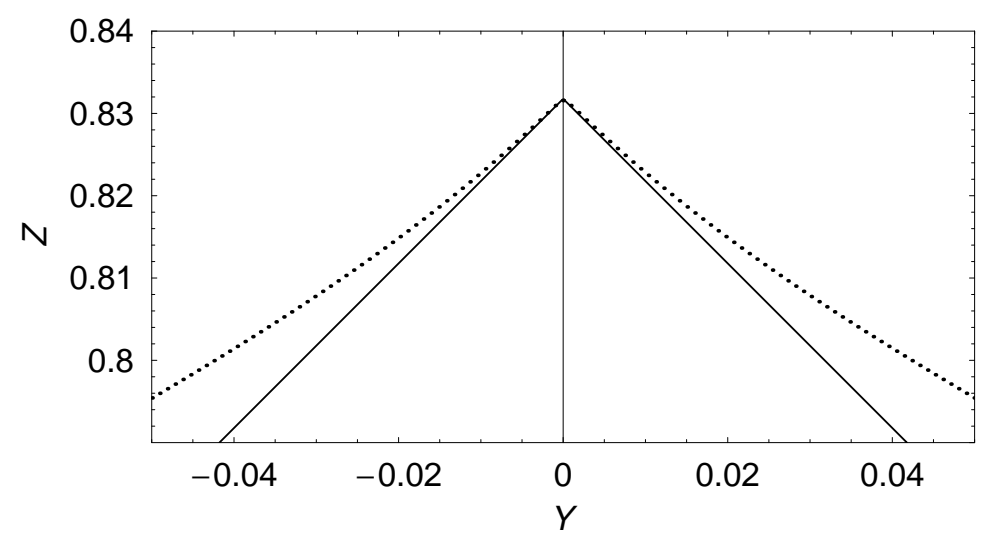

FIGURE 3. Almost-limiting wave profile near the crest in the case $\theta=0^{\circ}$ for $A_{c}=0.9986, N=25$ and $M=2^{8}: \cdots$, the numerical result; 一, the comparative profile $H(Y, 0)=H(0,0)-|Y|$ with an enclosed crest angle of $90^{\circ}$.

series (3.1), although the wave profile has a sharp crest. The Fourier expansion is generally incompatible with sharp crests. However, in this case it is compatible because the crest is a saddle point for the pressure distribution and the contour line for the pressure has a cusp at the saddle point (Okamura (1998)). In the case $\theta=0^{\circ}, A_{c}=0.9986, N=25$ and $M=2^{8}$, the maximum wave steepness $\epsilon_{\max }$ is 0.6280 , which is consistent with that of 0.6272 for standing waves in the case $A_{c}=0.9998, N=30$ and $M=2^{9}$ (Okamura (2003)). This agreement is not surprising because the two methods are essentially the same.

Figure 4 shows the almost-limiting wave profile near the crest with a maximum wave steepness of 0.4414 in the case $\theta=90^{\circ}, V_{c}=0.9630$ and $N=32$. The enclosed crest angle is likely to be $120^{\circ}$, although the crest is not sharp but rounded. We cannot obtain the limiting wave with a sharp corner using the present method because we expand the velocity potential $\Phi$ with a singular point at the crest in a Fourier series (3.3). This is a shortcoming of the present method. We can, however, obtain a maximum wave steepness of 0.4414 , which is consistent with 0.44316 for the limiting two-dimensional progressive wave (Williams (1981)). Therefore, the present method is useful for obtaining the almostlimiting wave profile (except in the neighbourhood of the crest) and for evaluating the maximum wave steepness of two-dimensional progressive waves.

It is important to note that (i) the crest is a singular point for the limiting progressive wave, whereas it is a saddle point in the pressure distribution for the limiting standing wave and (ii) only the 25th or 32nd order solution, which is expanded in a Fourier series in physical space without mapping, accurately expresses almost-limiting waves, including two-dimensional progressive waves.

\section{Numerical results}

\subsection{Maximum wave steepness and harmonic resonance}

We examine the maximum wave steepness $\epsilon_{\max }$ for various incident angles $\theta$. Figure 5 shows the present result for the maximum wave steepness as well as that obtained by Roberts (1983), who estimated the maximum wave steepness from the poles of Padé approximants. His result is qualitatively consistent with the present one, which is a little surprising because his method cannot treat harmonic resonance. There seems to be two gaps caused by harmonic resonance: one is around $\theta=12^{\circ}$ and the other is around 


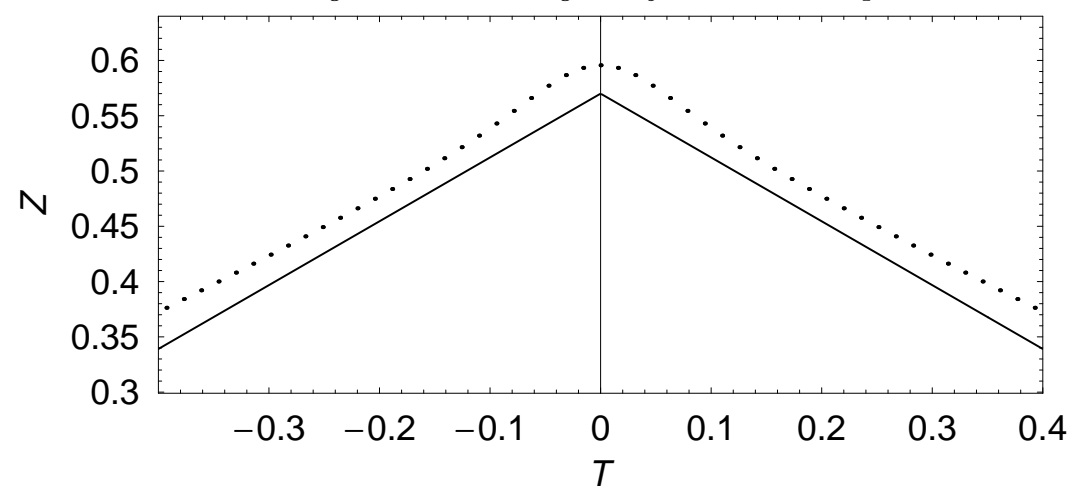

FiguRE 4 . Limiting wave profile near the crest in the case $\theta=90^{\circ}$ for $V_{c}=1, N=32: \cdots$, the numerical result; - , the comparative profile $H(T)=0.57-|T| / \sqrt{3}$ with an enclosed crest angle of $120^{\circ}$.

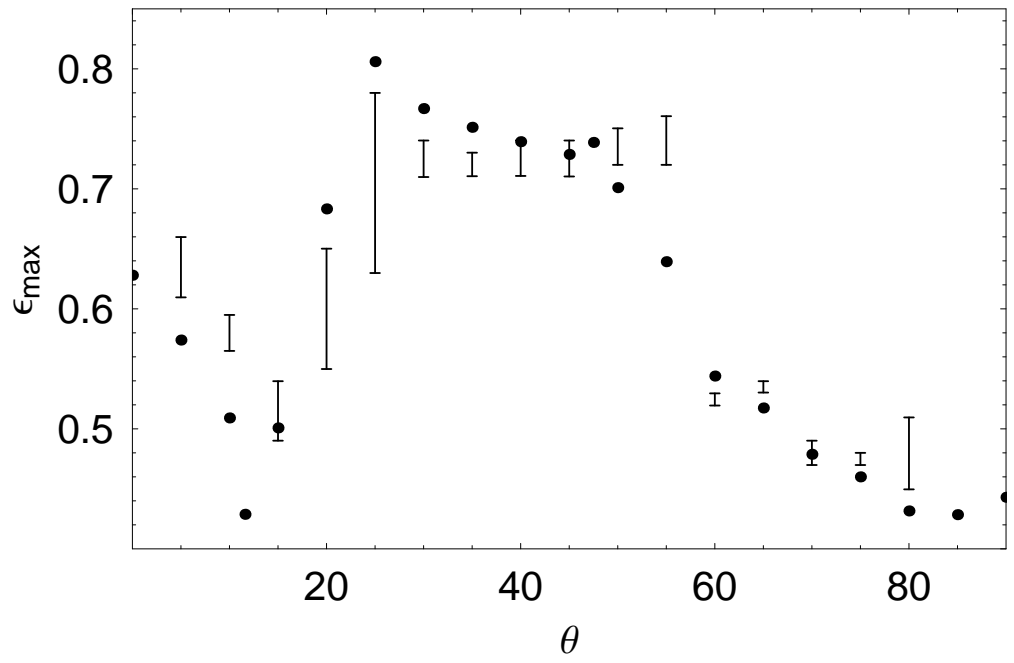

FIgURE 5. Dependence of the maximum wave steepness $\epsilon_{\max }$ on the incident angle $\theta$ for $N=25$ and $M=2^{8}$, except for the case $\theta=90^{\circ}$ for $N=32: \bullet$, the present result; I, Roberts (1983).

$\theta=55^{\circ}$. Harmonic resonance occurs if the linear wave frequency $\Omega\left(k_{x}, k_{y}\right)$ of a shortcrested wave satisfies

$$
\Omega\left(j k_{x}, l k_{y}\right)=j \Omega\left(k_{x}, k_{y}\right),
$$

where $\Omega\left(k_{x}, k_{y}\right)=g\left(k_{x}^{2}+k_{y}^{2}\right)^{1 / 4}$ is the linear dispersion relation (Roberts (1983)). In linear wave theory, (5.1) implies that the wave frequency of the $(j, l)$ th harmonic mode coincides with $j$ times that of the fundamental mode. Three typical resonant angles are

$$
\theta_{c}=0^{\circ} \quad \text { for } \quad A_{42}, \quad \theta_{c}=52.2^{\circ} \quad \text { for } \quad A_{62}, \quad \theta_{c}=36.7^{\circ} \text { for } A_{11,3} .
$$

There is a peak at $\theta=25^{\circ}$ and hence the maximum wave steepness for $\theta=25^{\circ}$ is the largest for all incident angles $\theta$, and its value is 1.8 times that of a two-dimensional progressive wave.

Table 2 lists the maximum wave steepness $\epsilon_{\max }$, the maximum effective wave steepness $\epsilon_{\max }^{*}$, the surface fluid acceleration $A_{c}$, the downward crest fluid acceleration $A_{c}^{*}$, the surface velocity $V_{c}$ and the crest velocity $V_{c}^{*}$ for various incident angles $\theta$. The following results can be obtained from this table. First, the maximum wave steepness $\epsilon_{\max }$ differs 


\begin{tabular}{lcccccccccc}
\hline & & & & & & & & & & \\
$\theta$ & 0 & 10 & 10 & 25 & 40 & 47.5 & 50 & 70 & 85 & 90 \\
$\epsilon_{\max }$ & 0.6280 & 0.5091 & $(0.3265)$ & 0.8060 & 0.7393 & 0.7387 & 0.7010 & 0.4788 & 0.4373 & 0.4414 \\
$\epsilon_{\max }^{*}$ & 0.6280 & 0.5091 & $(0.3441)$ & 0.8066 & 0.7410 & 0.7387 & 0.7010 & 0.4831 & 0.6702 & 0.4414 \\
$A_{c}$ & 0.9986 & $(0.7603)$ & 0.9910 & 0.9942 & 0.9781 & 0.9993 & 0.6883 & 0.6804 & 0.4842 & 0.6135 \\
$A_{c}^{*}$ & 0.9986 & $(0.7603)$ & 0.4844 & 0.9823 & 0.8453 & 0.2051 & 0.2972 & 0.0667 & 0.0477 & 0.1545 \\
$V_{c}$ & 0 & 0.0201 & 0.0119 & 0.2083 & 0.5331 & 0.9660 & 0.9468 & 0.9871 & 0.9903 & 0.9630 \\
$V_{c}^{*}$ & 0 & 0.0201 & 0.0108 & 0.2076 & 0.5189 & 0.9660 & 0.9468 & 0.9871 & 0.9903 & 0.9630
\end{tabular}

TABLE 2. The maximum wave steepness $\epsilon_{\max }$, maximum effective wave steepness $\epsilon_{\max }^{*}$, surface fluid acceleration $A_{c}$, downward crest fluid acceleration $A_{c}^{*}$, surface velocity $V_{c}$ and crest velocity $V_{c}^{*}$ for various incident angles $\theta . N=25, M=2^{8}$ for $0^{\circ} \leqslant \theta \leqslant 85^{\circ} ; N=32$ for $\theta=90^{\circ}$.

little from the maximum effective wave steepness $\epsilon_{\max }^{*}$ for $\theta=10^{\circ}, 25^{\circ}, 40^{\circ}$ and $70^{\circ}$ because there is a possibility that $\epsilon_{\max }$ is not the maximum wave steepness for resonant waves, as has already been explained in the discussion following (2.9). Second, limiting waves with the maximum wave steepness satisfy the limiting conditions $A_{c}=1$ and $V_{c}=1$ approximately for $0^{\circ} \leqslant \theta \leqslant 47.5^{\circ}$ and $47.5^{\circ} \leqslant \theta \leqslant 90^{\circ}$ respectively, except for $\theta=10^{\circ}$. Third, $A_{c} \neq A_{c}^{*}$ except for $\theta=0^{\circ}$ and the left column of $\theta=10^{\circ}$, while $V_{c}=V_{c}^{*}$ for $47.5^{\circ} \leqslant \theta \leqslant 90^{\circ}$. This means that the limiting condition of the short-crested wave is inconsistent with that of the standing wave (2.11) but is consistent with the generalised condition (2.14) in the case $0^{\circ}<\theta \leqslant 47.5^{\circ}$, while it is consistent with that of the two-dimensional progressive wave $(2.13)$ in the case $47.5^{\circ} \leqslant \theta<90^{\circ}$.

Figure 6 shows four bifurcation diagrams in the $\left(A_{11}, A_{42}\right)$-plane. This bifurcation, which is related to harmonic resonance for $\theta_{c}=0^{\circ}$, is supercritical. The bifurcation point shifts to the right as $\theta$ increases, while it approaches the origin as $\theta \rightarrow 0^{\circ}$, as expected. The closed circle and the open square denote the almost-limiting wave with $A_{c} \approx 1$ and the highest wave with the maximum wave steepness respectively, and an endpoint without any symbol corresponds to neither the almost-limiting nor the highest wave. For example, let us consider the case $\theta=11.6^{\circ}$ shown in figure $6(\mathrm{c})$. The surface fluid acceleration $A_{c}$ is 0.5245 at the endpoint without any symbol, $\left(A_{11}, A_{42}\right)=(0.338,-0.0084)$, on the lower branch. This acceleration is nearly half that in the limiting wave, while there is no limiting wave on the loop branch. Thus, we cannot obtain an almost-limiting wave in the case $\theta=11.6^{\circ}$ in the present calculation, although the endpoint of the lower branch may correspond to the limiting wave in the case $\theta=10^{\circ}$. The reason for this is currently unknown. In the case $\theta=7^{\circ}$, as $A_{11}$ increases just beyond the bifurcation point, the supercritical bifurcation gives rise to three solutions: two resonant branches with a larger value of $\left|A_{42}\right|$ and one non-resonant branch with a smaller value of $\left|A_{42}\right|$. The highest wave $(\square)$ does not coincide with the almost-limiting wave with $A_{c} \approx 1(\bullet)$ in this case, as well as in the case $\theta=10^{\circ}$. The data for these waves in the case $\theta=10^{\circ}$ are listed in table 2. The left and right columns for $\theta=10^{\circ}$ in this table correspond to a non-resonant highest wave with a smaller value of $A_{c}$ and a resonant almost-limiting wave with a smaller value of $\epsilon_{\max }$. The value in the round brackets is smaller than the corresponding value in the other column. The endpoints of the resonant and non-resonant branches approach each other as $\theta$ increases from $7^{\circ}$, and the two branches nearly form a loop without the limiting wave at $\theta=10^{\circ}$. It becomes a true loop with a little increase in $\theta$ and this loop becomes smaller as $\theta$ increases. Finally, it disappears in the case $\theta=11.7^{\circ}$. The disappearance of the loop can be seen in figures $6(\mathrm{~b})-(\mathrm{d})$. The harmonic resonance related to $A_{42}$ disturbs the formation of the limiting wave and hence the maximum wave steepness decreases as $\theta$ increases for $0^{\circ} \leqslant \theta \lesssim 12^{\circ}$ as shown in figure 5 . The maximum 

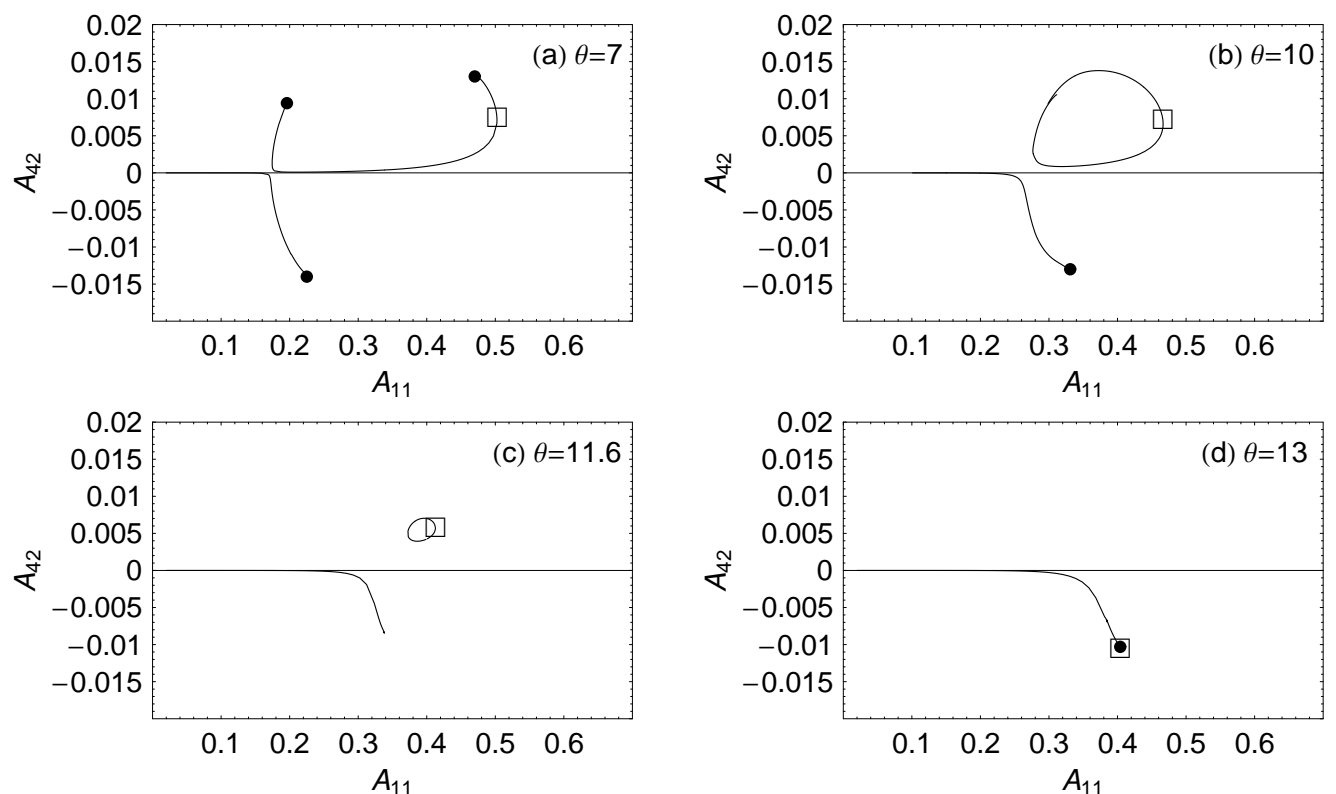

FiguRE 6 . Four bifurcation diagrams in the $\left(A_{11}, A_{42}\right)$-plane: (a) $\theta=7^{\circ}$; (b) $\theta=10^{\circ}$; (c) $\theta=11.6^{\circ}$; (d) $\theta=13^{\circ}$. $\bullet$ : the almost-limiting wave with $A_{c} \approx 1 ; \square$ : the highest wave with $\epsilon=\epsilon_{\max }$.

wave steepness increases rapidly as $\theta$ increases beyond about $12^{\circ}$ because of the small influence of harmonic resonance. Therefore, there is a gap around $\theta=12^{\circ}$.

Figure 7 shows four bifurcation diagrams in the $\left(A_{11}, A_{62}\right)$-plane. This bifurcation, which is related to harmonic resonance for $\theta_{c}=52.2^{\circ}$, is subcritical. Figures $7(\mathrm{a})$ and (b) confirm that this bifurcation occurs for $\theta>\theta_{c}$. In the case $\theta=55^{\circ}$, the almostlimiting wave with $A_{c} \approx 1(\bullet)$ is a resonant wave, while the highest and almost-limiting wave with $\epsilon=\epsilon_{\max }$ and $V_{c} \approx 1$ (回) is a non-resonant wave. In the case $\theta=60^{\circ}$, the bifurcation point is near the point related to the highest wave or the almost-limiting wave and hence the harmonic resonance disturbs the formation of the highest wave and the limiting wave. However, the harmonic resonance related to $A_{62}$ does not affect the formation of the limiting wave except for $\theta=60^{\circ}$, because the non-resonant branch for large $A_{11}$ is distinct from the two resonant branches in the subcritical bifurcation unlike the cases $\theta=7^{\circ}, 10^{\circ}$ in the supercritical bifurcation. Thus the non-resonant branch and any one of the resonant branches never form a loop. Therefore, there must be no gap around $\theta=55^{\circ}$. This statement may appear to be incompatible with the result displayed in Fig. 5. To check this, we investigate the maximum actual wave steepness $\tilde{\epsilon}_{\max }$ defined in (2.10) instead of the maximum wave steepness $\epsilon_{\max }$. Figure 8 shows the dependence of $\tilde{\epsilon}_{\max }$ on $\theta$. This figure indicates that the gap around $\theta=12^{\circ}$ still exists, whereas the gap around $\theta=55^{\circ}$ disappears.

In figure $7(\mathrm{a})$, the branch is cut off with a small distance near $A_{11}=0.5$. This cutoff is due to harmonic resonance $A_{11,3}$, as shown in figure 9 . There are very many (probably infinite) solutions due to harmonic resonance for given values of $\theta$ and $\epsilon$ (Roberts (1983)). However, as the resonance moves to higher harmonics, it becomes much weaker. Hence, we cannot distinguish between the resonant branch and the non-resonant branch for higher harmonic resonance, as shown in figures 7(a) and 9(a). Therefore, higher harmonic resonance is not so important. 

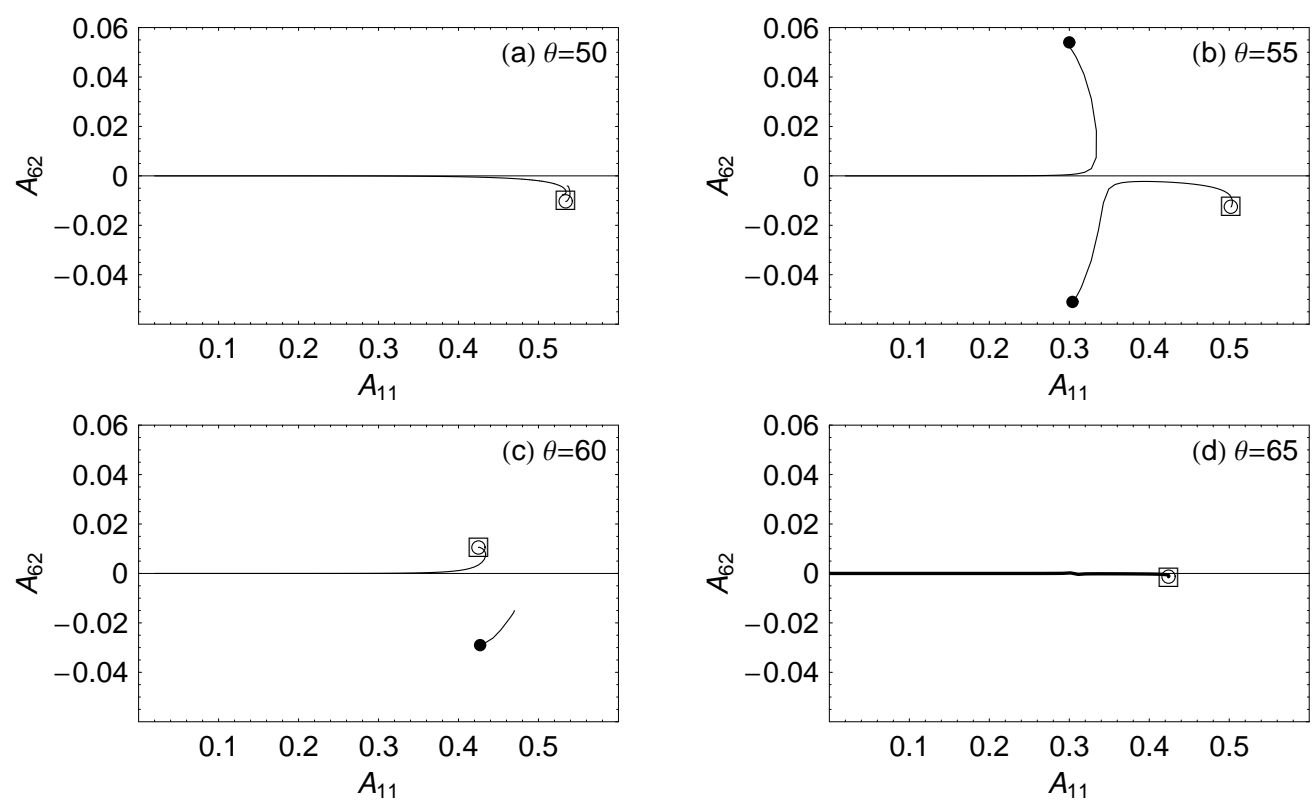

Figure 7 . Four bifurcation diagrams in the $\left(A_{11}, A_{62}\right)$-plane: (a) $\theta=50^{\circ}$; (b) $\theta=55^{\circ}$; (c) $\theta=60^{\circ}$; (d) $\theta=65^{\circ}$. $\bullet$ : the almost-limiting wave with $A_{c} \approx 1$; ०: the almost-limiting wave with $V_{c} \approx 1 ; \square:$ the highest wave with $\epsilon=\epsilon_{\max }$.

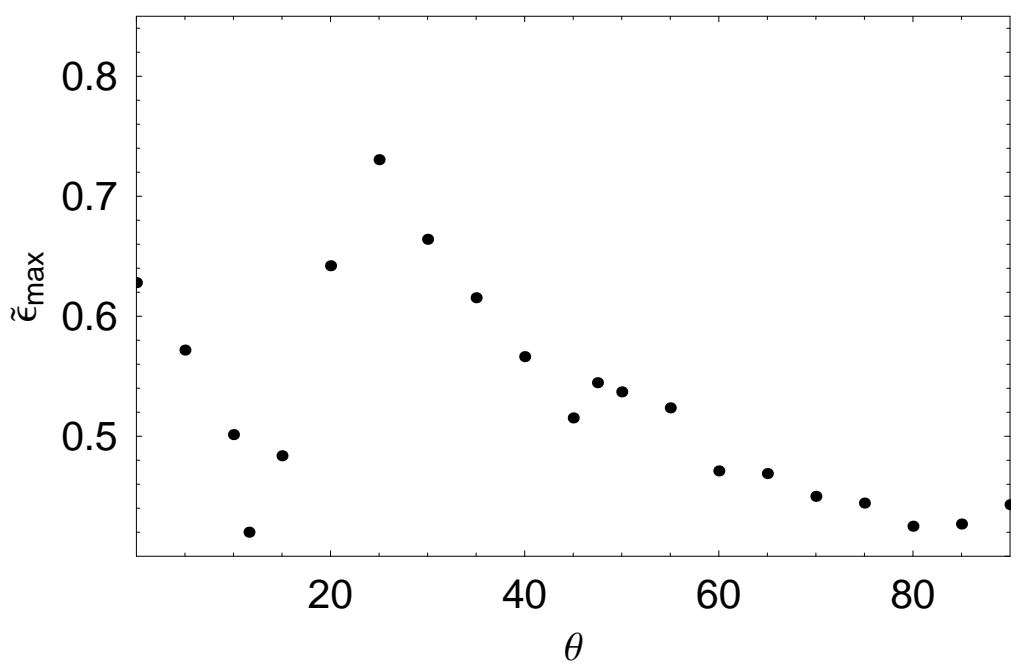

FiguRE 8. Dependence of the maximum actual wave steepness $\tilde{\epsilon}_{\max }$, defined in (2.10), on the incident angle $\theta$. These data are the same as those in figure 5 .

\subsection{Limiting wave profiles}

We investigate the limiting profiles of short-crested waves for various incident angles $\theta$. In general, in the case $0^{\circ} \leqslant \theta \leqslant 47.5^{\circ}$, the short-crested wave has the limiting wave profile with $A_{c}=1$ given in (2.14), which corresponds to the limiting condition of the standing wave, while in the case $47.5^{\circ} \leqslant \theta \leqslant 90^{\circ}$, the short-crested wave has the limiting wave profile with $V_{c}=1$ given in $(2.15)$, which corresponds to the limiting condition 

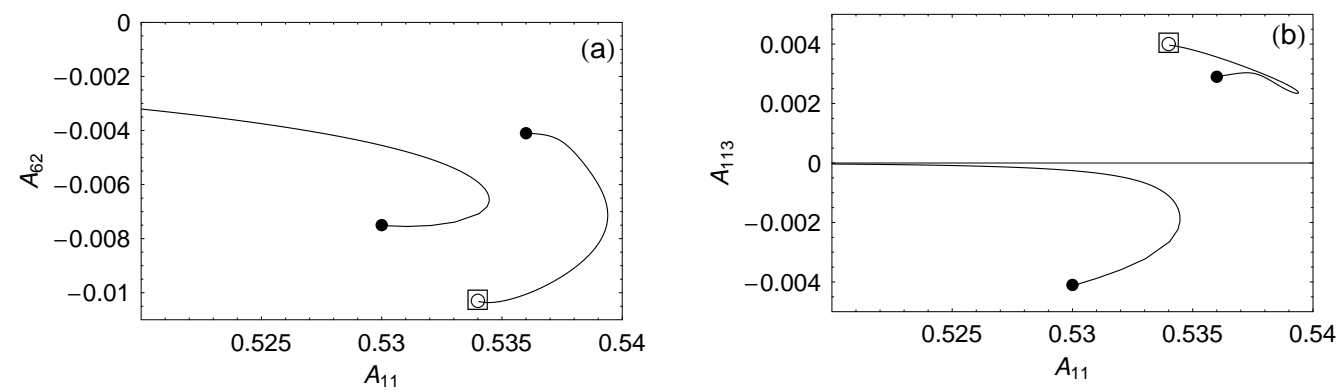

FiguRE 9. (a) Enlargement of the bifurcation diagram in the $\left(A_{11}, A_{62}\right)$-plane shown in figure $7(\mathrm{a})$. (b) The corresponding bifurcation diagram in the $\left(A_{11}, A_{11,3}\right)$-plane. $\bullet$ : the almost-limiting wave with $A_{c} \approx 1$; ०: the almost-limiting wave with $V_{c} \approx 1 ; \square$ : the highest wave with $\epsilon=\epsilon_{\max }$

of the two-dimensional progressive wave. The enclosed crest angles are $90^{\circ}$ and $120^{\circ}$ for $0^{\circ} \leqslant \theta \leqslant 47.5^{\circ}$ and $47.5^{\circ} \leqslant \theta \leqslant 90^{\circ}$, respectively.

Figures 10 and 11 show, in the spatial coordinates $\left(x^{*}, y^{*}, z^{*}\right)$ instead of $(T, Y, Z)$, the almost-limiting wave profiles at $t^{*}=0$ with $A_{c}=0.9856$ and 0.9756 respectively, for $\theta=7^{\circ}$. These profiles correspond to the resonant wave profiles at the points $\left(A_{11}, A_{42}\right)=$ $(0.195,0.0092)$ and $\left(A_{11}, A_{42}\right)=(0.225,-0.0137)$ on the resonant branches in the bifurcation diagram shown in figure $6(\mathrm{a})$. It is not possible to clearly detect any sharp crest in figure 10(a) and 11(a) at low resolution, although the profile in figure 10(a) has a rugged surface. Figures 10(b) and 11(b) show detailed cross-sectional views, which reveal sharp corners. There are $90^{\circ}$-sharp corners at the points $\left(y^{*}, z^{*}\right)=( \pm 0.301,0.253)$ in figure 10(b) and $\left(y^{*}, z^{*}\right)=( \pm 0.624,0.254)$ in figure $11(\mathrm{~b})$ with $A_{c}=0.9856, A_{c}^{*}=0.1645$ and $A_{c}=0.9765, A_{c}^{*}=0.4766$, respectively. Note that the surface fluid acceleration $A_{c}$ is not directed downward and the downward crest fluid acceleration $A_{c}^{*}$ is much smaller than the limiting value of 1 . The crest shown in figure 11 is flatter than that shown in figure 10, while the trough shown in figure 10 is flatter than that shown in figure 11, because the crest becomes flatter for $A_{42}<0$ and the trough becomes flatter for $A_{42}>0$ for resonant waves, as shown in figure 1. In other words, the crest becomes flatter than the trough for resonant waves if the sign of the fundamental mode $A_{11}$ differs from that of its harmonic resonant mode $A_{42}$.

Figure 12 shows the almost-limiting wave profile at $t^{*}=0$ with $A_{c}=0.9942$ for $\theta=25^{\circ}$. A perspective view is shown in figure $12(\mathrm{a})$ and three cross-sectional views are shown in figures 12(b)-(d). We cannot detect any sharp crest in figure 12(a) at low resolution. Figure 12(c) shows the cross-sectional view, $x^{*}=0$, of the profile near the peak at high resolution. The crest is certainly rounded even in the high resolution plot. Figure 12(b) shows the cross-sectional view, $y^{*}=0$, of the profile near the peak at high resolution. The wave profile reaches the maximum not at the point, $\left(x^{*}, y^{*}\right)=(0,0)$, but at $\left(x^{*}, y^{*}\right)=( \pm 0.116,0)$. We next examine the cross-sectional view, $x^{*}=0.116$, of the profile near the peak at high resolution shown in figure 12(d). The enclosed crest angle is $90^{\circ}$ and the direction of the surface fluid acceleration $A_{c}$ is downward. Therefore, the enclosed crest angle is $90^{\circ}$ at the point where the wave profile reaches its maximum.

Figure 13 shows the almost-limiting wave profile at $t^{*}=0$ with $A_{c}=0.9781$ for $\theta=40^{\circ}$. A perspective view is shown in figure 13(a), and two cross-sectional views are shown in figures 13(b) and (c). There seems to be a sharp corner at the crest of the limiting wave profile in figure 13(a) at low resolution. Figure 13(b) shows the crosssectional view, $y^{*}=0$, of the profile near the peak at high resolution. The wave profile 
(a)
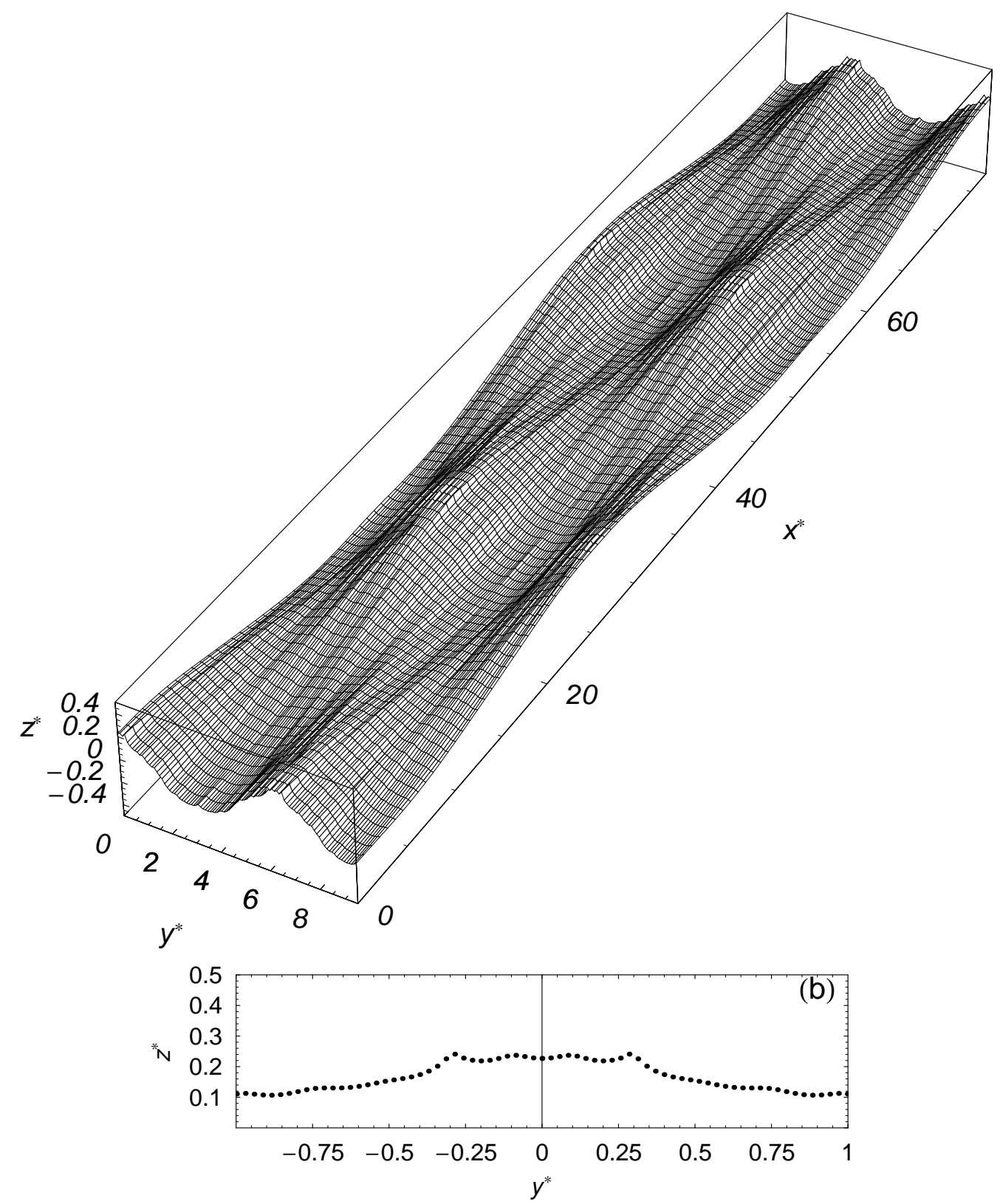

Figure 10. (a) Almost-limiting wave profile with $A_{c}=0.9856$ for $\theta=7^{\circ}$, corresponding to the point $\left(A_{11}, A_{42}\right)=(0.195,0.0092)$ in the bifurcation diagram shown in figure $6(\mathrm{a})$. (b) Cross-sectional view, $x^{*}=0$, of the wave profile near the peak.

reaches its maximum at the point $\left(x^{*}, y^{*}\right)=(0,0)$ even at high resolution. However, in the cross-sectional view, $x^{*}=0$, shown in figure 13(c), the free surface forms a sharp corner not at the point, $\left(x^{*}, y^{*}\right)=(0,0)$, but at the points, $\left(x^{*}, y^{*}\right)=(0, \pm 0.0030)$, and the crest angle may be $90^{\circ}$. The surface fluid acceleration $A_{c}$ reaches its maximum value 
(a)
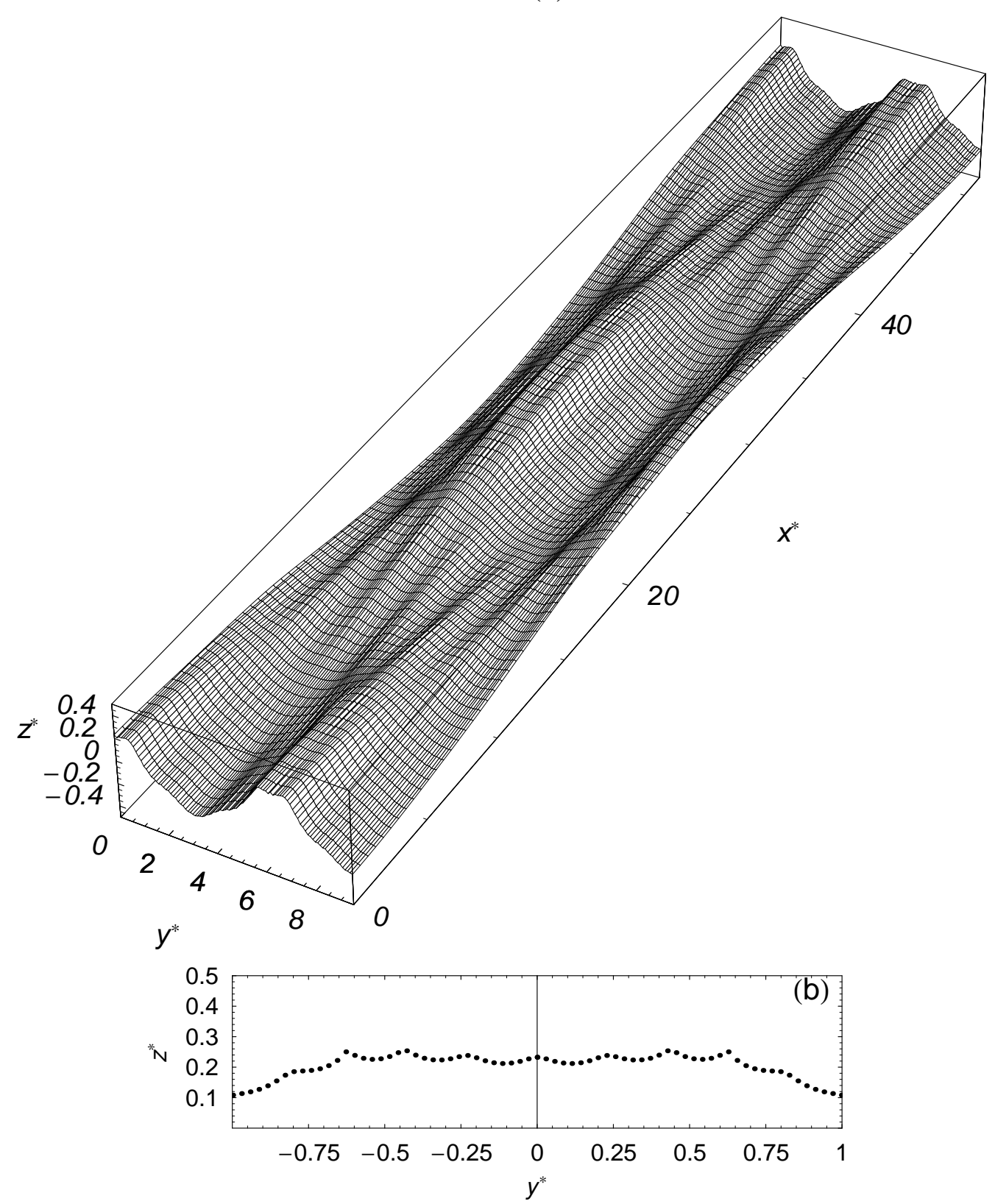

Figure 11. (a) Almost-limiting wave profile with $A_{c}=0.9756$ for $\theta=7^{\circ}$, corresponding to the point $\left(A_{11}, A_{42}\right)=(0.225,-0.0137)$ in the bifurcation diagram shown in figure $6(\mathrm{a})$. (b) Cross-sectional view, $x^{*}=0$, of the wave profile near the peak.

of 0.9781 at the corner and its direction is not downward. The surface profile is nearly flat near the point, $\left(x^{*}, y^{*}\right)=(0,0)$.

Figure 14 shows the almost-limiting wave profile at $t^{*}=0$ with $A_{c}=0.9993$ and $V_{c}=$ 0.9660 for $\theta=47.5^{\circ}$. A perspective view is shown in figure 14(a) and two cross-sectional views are shown in figures $14(\mathrm{~b})$ and (c). In this case, the limiting conditions (2.14) and 
(a)
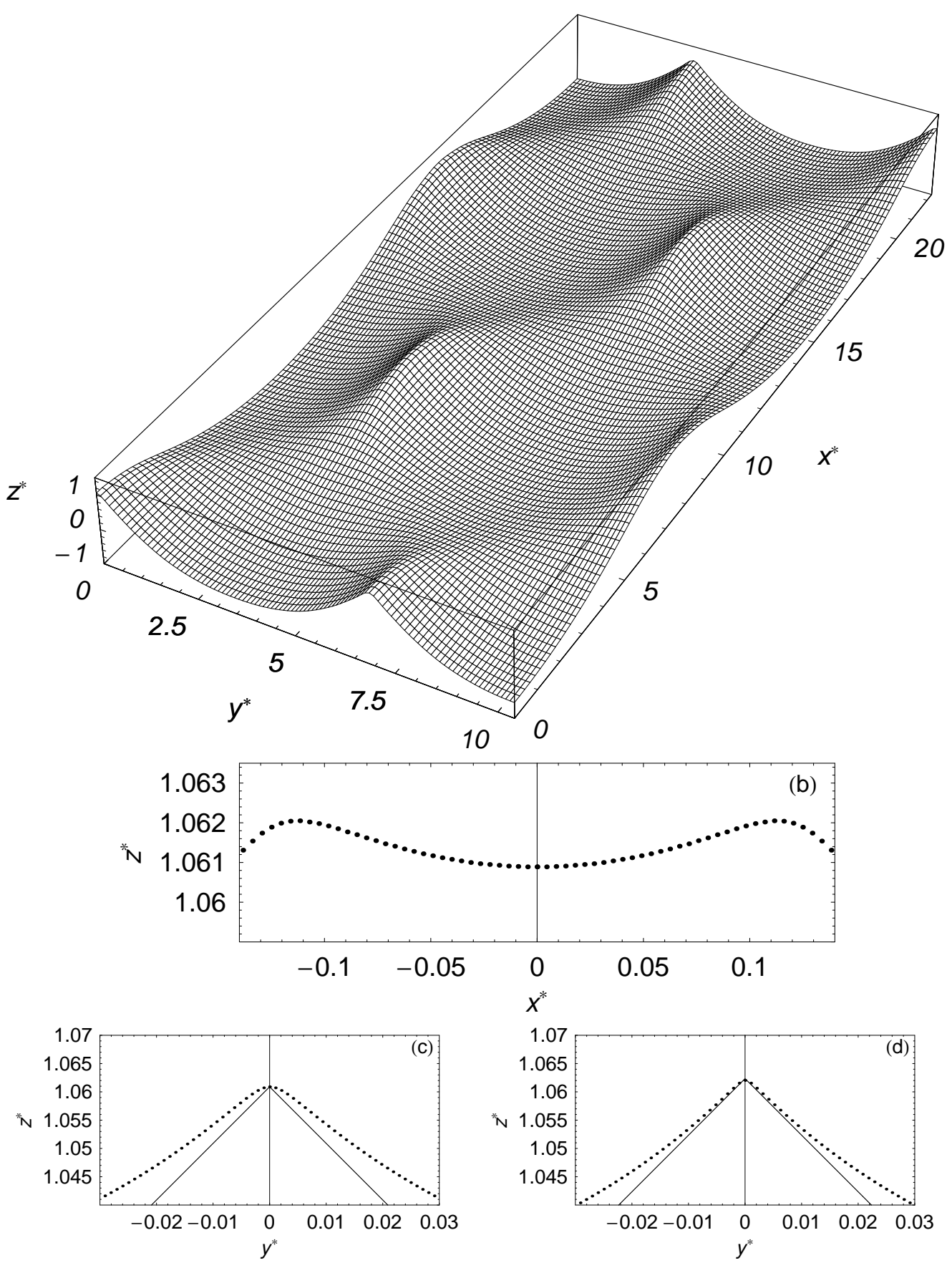

Figure 12. (a) Almost-limiting wave profile with $A_{c}=0.9942$ for $\theta=25^{\circ}$. (b) Cross-sectional view, $y^{*}=0$, of the wave profile near the peak. (c) Cross-sectional view, $x^{*}=0$, of the wave profile near the peak together with a comparative profile $H^{*}\left(0, y^{*}, 0\right)=H^{*}(0,0,0)-\left|y^{*}\right|$ with an enclosed crest angle of $90^{\circ}$. (d) Cross-sectional view, $x^{*}=0.116$, of the wave profile near the peak together with a comparative profile $H^{*}\left(0, y^{*}, 0\right)=H^{*}(0,0,0)-\left|y^{*}\right|$ with an enclosed crest angle of $90^{\circ}$. 
(a)
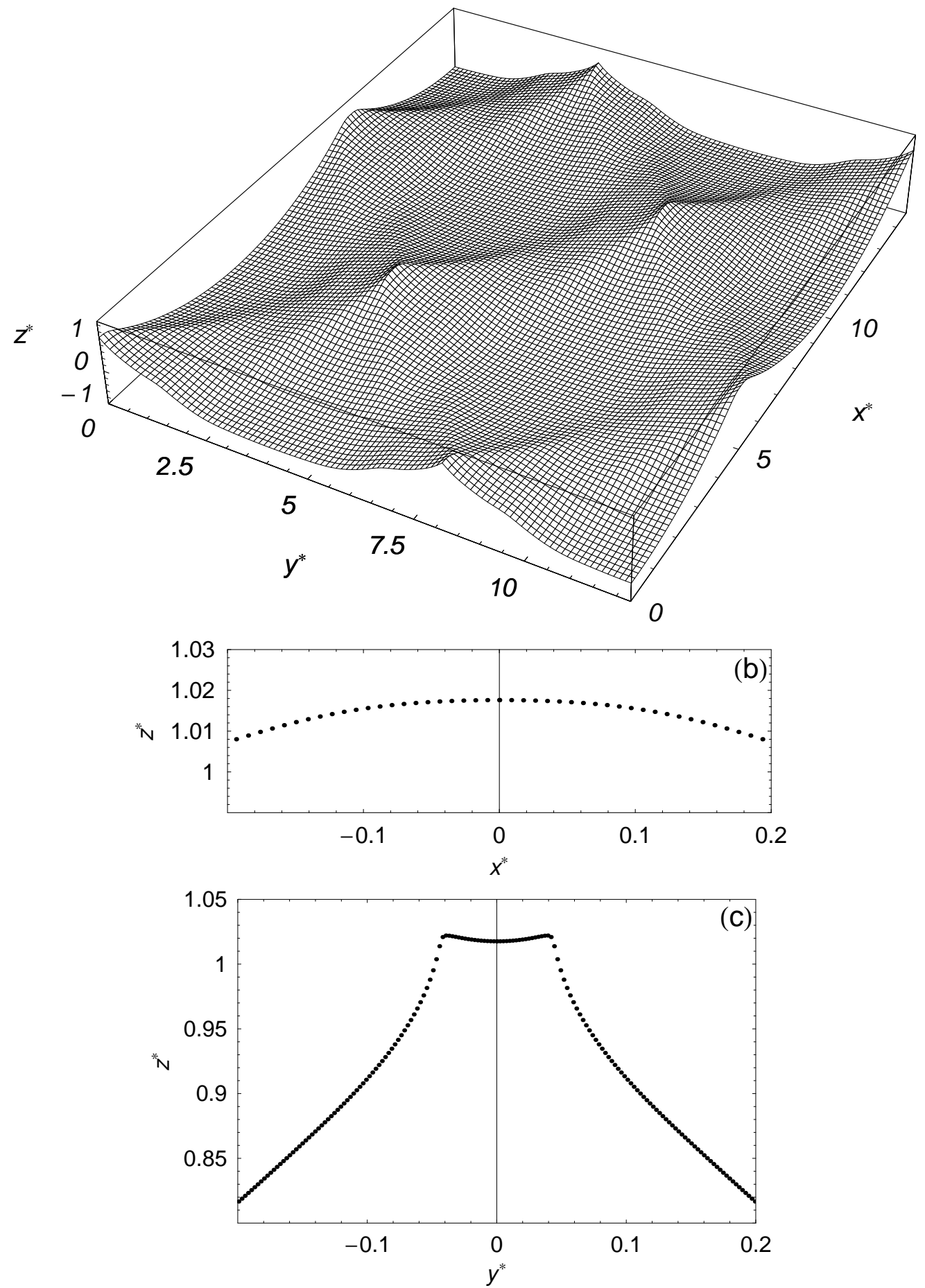

Figure 13. (a) Almost-limiting wave profile with $A_{c}=0.9781$ for $\theta=40^{\circ}$. (b) Cross-sectional view, $y^{*}=0$, of the wave profile near the peak. (c) Cross-sectional view, $x^{*}=0$, of the wave profile near the peak. 
(2.15) are simultaneously satisfied, and then we can detect two enclosed crest angles, $90^{\circ}$ and $120^{\circ}$, in the limiting wave profile, as shown in figure 14 (a) at low resolution. Figure 14(b) at high resolution suggests that the enclosed crest angle is $120^{\circ}$ in the cross-section, $y^{*}=0$. The shape of the rounded crest is false because of a shortcoming in the present method, as shown in figure 4 . The shape of the profile near the crest in figure 14(c) is wavy but may also be false because of a shortcoming in the present method; the limiting shape should probably be similar to that in figure 13(c).

Figure 15 shows the almost-limiting wave profile at $t^{*}=0$ with $V_{c}=0.9871$ for $\theta=70^{\circ}$. A perspective view is shown in figure 15 (a), and two cross-sectional views, $y^{*}=0$ and $x^{*}=0$, are shown in figures $15(\mathrm{~b})$ and (c). Note that the limiting condition is $V_{c}=1$ given in (2.15) for $\theta \geqslant 47.5^{\circ}$. Figure 15(b) suggests that the enclosed crest angle is $120^{\circ}$ in the cross-section $y^{*}=0$, although the crest is slightly rounded because of a shortcoming in the present method.

Figure 16 shows the almost-limiting wave profile at $t^{*}=0$ with $V_{c}=0.9903$ for $\theta=85^{\circ}$. A perspective view is shown in figure 16 (a), and a cross-sectional view, $y^{*}=0$, is shown in figures 16(b). Figure 16(b) suggests that the enclosed crest angle is $120^{\circ}$ in the crosssection, $y^{*}=0$. This wave corresponds to a fully nonlinear version of the fourth-order long-crested waves obtained by Roberts \& Peregrine (1983).

Figure 17 shows the almost-limiting wave profile at $t^{*}=0$ with $V_{c}=0.9871$ for $\theta=55^{\circ}$ in the case of harmonic resonance. This wave corresponds to the point $\left(A_{11}, A_{62}\right)=$ $(0.296,0.0539)$ in the bifurcation diagram shown in figure $7(\mathrm{c})$. We can confirm that the wave contains the sixth harmonic mode $A_{62}$, which is the same order of magnitude as the fundamental mode $A_{11}$. The enclosed crest angle may be $90^{\circ}$.

\section{Summary and discussion}

We have clarified the characteristics of resonant short-crested waves by drawing bifurcation diagrams. In particular, the two main harmonic resonances related to $A_{42}$ and $A_{62}$ have been investigated in detail. The results are listed below.

(a) In general, the limiting wave is realized for $0^{\circ} \leqslant \theta \leqslant 47.5^{\circ}$ if the fluid particle acceleration is equal to the gravitational acceleration, $A_{c}=1$ given in (2.14), while the limiting wave is realized for $47.5^{\circ} \leqslant \theta \leqslant 90^{\circ}$ if the fluid particle velocity in the $x$-direction at the crest is equal to the phase velocity, $V_{c}=1$ given in (2.15). The enclosed crest angles are $90^{\circ}$ and $120^{\circ}$ for $0^{\circ} \leqslant \theta \leqslant 47.5^{\circ}$ and $47.5^{\circ} \leqslant \theta \leqslant 90^{\circ}$, respectively. Therefore, waves for $0^{\circ} \leqslant \theta \leqslant 47.5^{\circ}$ and $47.5^{\circ} \leqslant \theta \leqslant 90^{\circ}$ have the characteristics of standing waves and two-dimensional progressive waves, respectively.

(b) Two main harmonic resonances related to $A_{42}$ and $A_{62}$ undergo supercritical and subcritical bifurcations, respectively. In the bifurcation diagram related to $A_{42}$, a loop is formed by the resonant and non-resonant branches coalescing. The maximum wave steepness is reduced because of the formation of a loop around $\theta=12^{\circ}$.

(c) In the case of resonant waves, if the sign of the fundamental mode differs from that of its harmonic resonant mode, the crest is flatter than the trough. Otherwise, the crest is steeper than the trough, as is the case for non-resonant waves.

(d) The highest wave does not necessarily coincide with the limiting wave, such as $\theta=7^{\circ}$ and $10^{\circ}$.

(e) In the cases $0^{\circ} \leqslant \theta \leqslant 47.5^{\circ}$, a sharp corner does not necessarily appear at the crest, $x^{*}=y^{*}=0$. The surface fluid acceleration $A_{c}$ reaches the limiting value, $A_{c}=1$, at the corner and its direction is not downward. In the cases $47.5^{\circ} \leqslant \theta \leqslant 90^{\circ}$, a sharp corner appears at the crest, $x^{*}=y^{*}=0$. The crest velocity $V_{c}$ reaches the limiting value, 
(a)
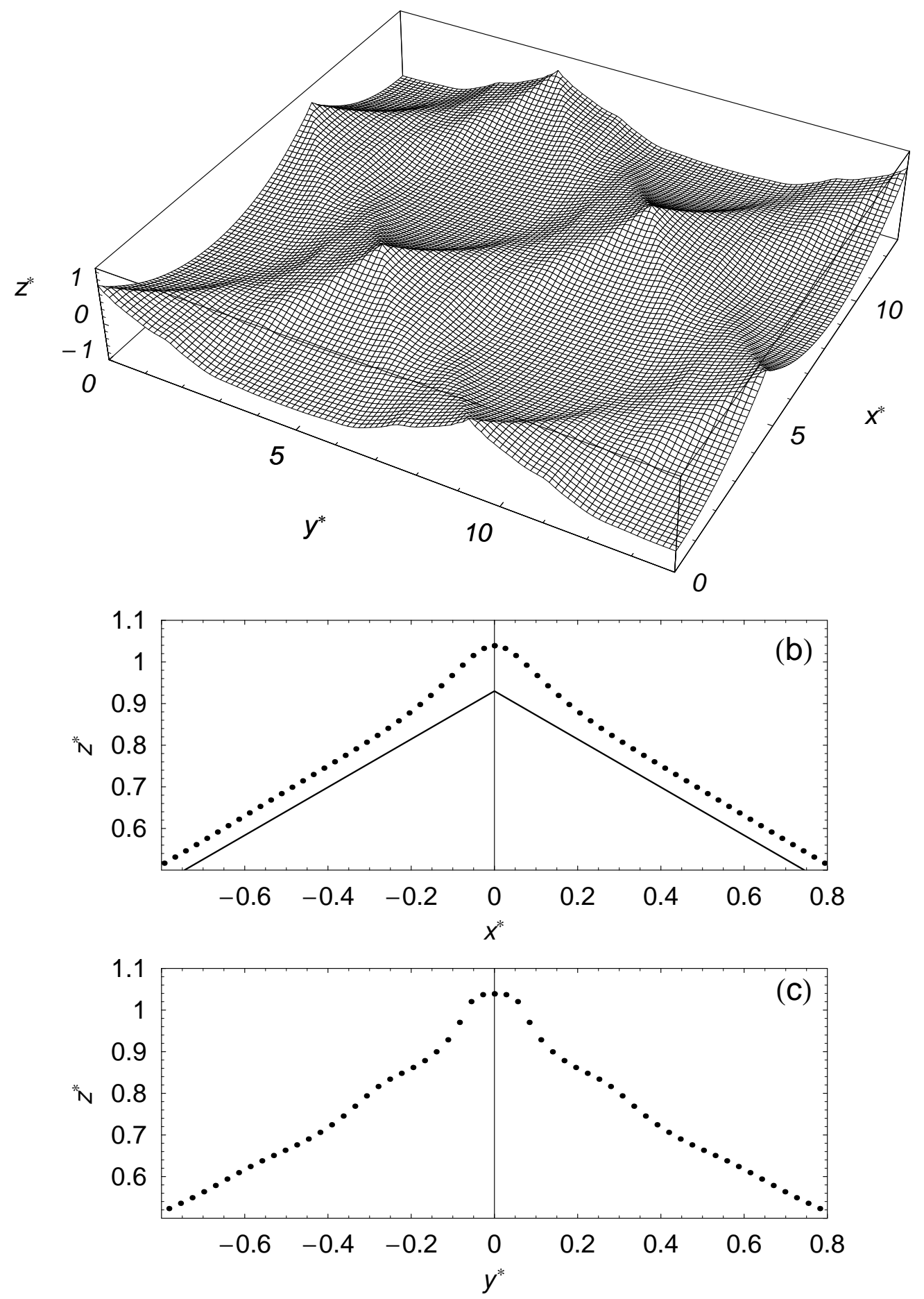

FiguRE 14. (a) Almost-limiting wave profile with $A_{c}=0.9993$ and $V_{c}=0.9660$ for $\theta=47.5^{\circ}$. (b) Cross-sectional view, $y^{*}=0$, of the wave profile near the peak: $\cdots$, the numerical result; the comparative profile $H^{*}\left(0, y^{*}, 0\right)=0.93-\left|y^{*}\right| / \sqrt{3}$ with an enclosed crest angle of $120^{\circ}$. (c) Cross-sectional view, $x^{*}=0$, of the wave profile near the peak. 
(a)
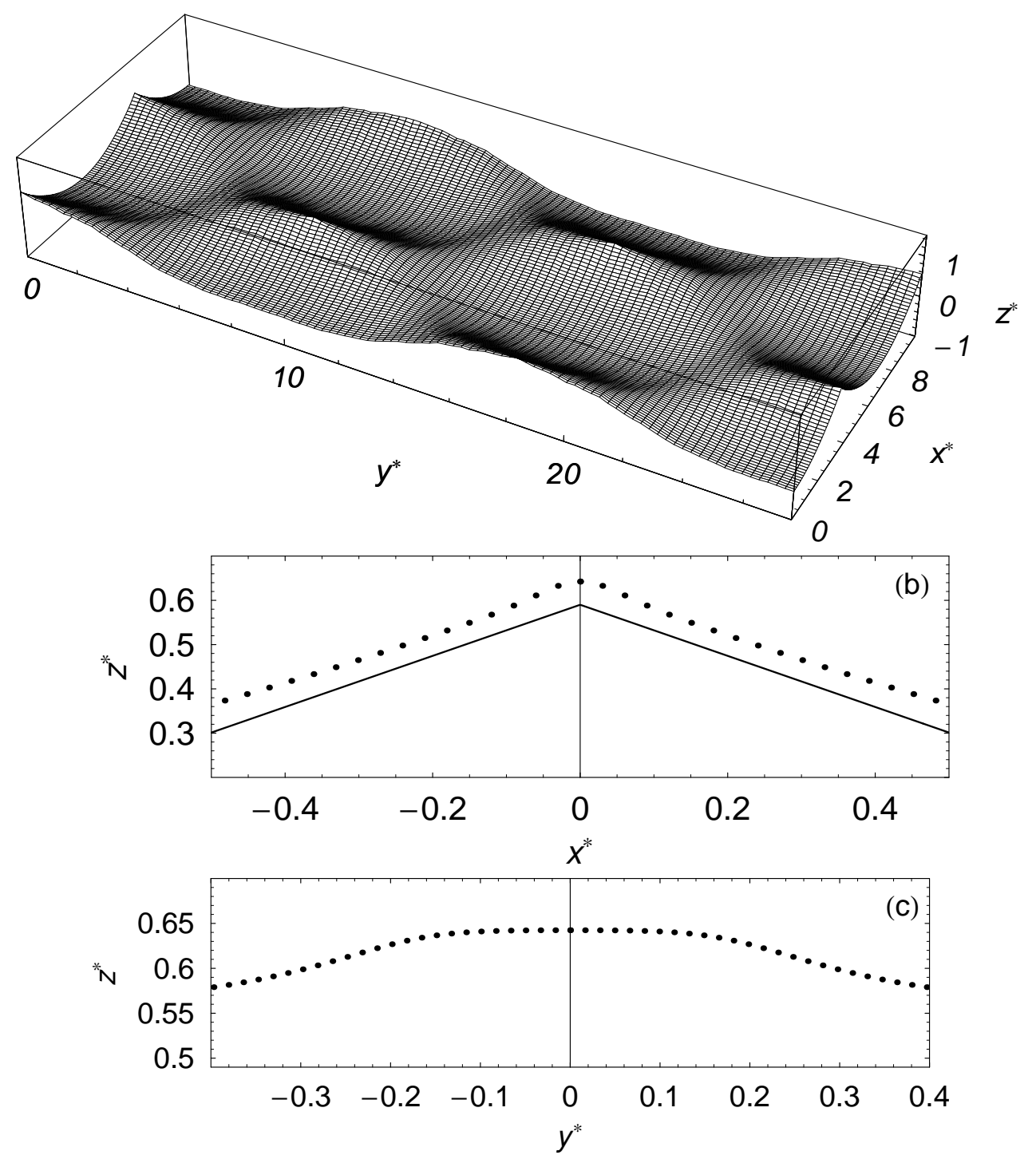

FiguRE 15. (a) Almost-limiting wave profile with $V_{c}=0.9871$ for $\theta=70^{\circ}$. (b) Cross-sectional view, $y^{*}=0$, of the wave profile near the peak: $\cdots$, the numerical result; - , the comparative profile $H^{*}\left(x^{*}, 0,0\right)=0.59-\left|x^{*}\right| / \sqrt{3}$ with an enclosed crest angle of $120^{\circ}$. (c) Cross-sectional view, $x^{*}=0$, of the wave profile near the peak.

$V_{c}=1$, at the corner, and the crest velocity is equal to the surface velocity, $V_{c}=V_{c}^{*}$, as in two-dimensional progressive waves.

The present method is a powerful method for calculating almost-limiting short-crested waves with $A_{c} \approx 1$, and then the wave profiles clearly show that the enclosed crest angle is $90^{\circ}$. Furthermore, the present method is also a powerful method for calculating almostlimiting short-crested waves with $V_{c} \approx 1$, and then we can obtain a reliable value for the maximum wave steepness. However, we cannot obtain the limiting wave profile with a sharp corner for $47.5^{\circ} \leqslant \theta \leqslant 90^{\circ}$ because the velocity potential $\Phi$ at $y^{*}=0$ is expressed 
(a)
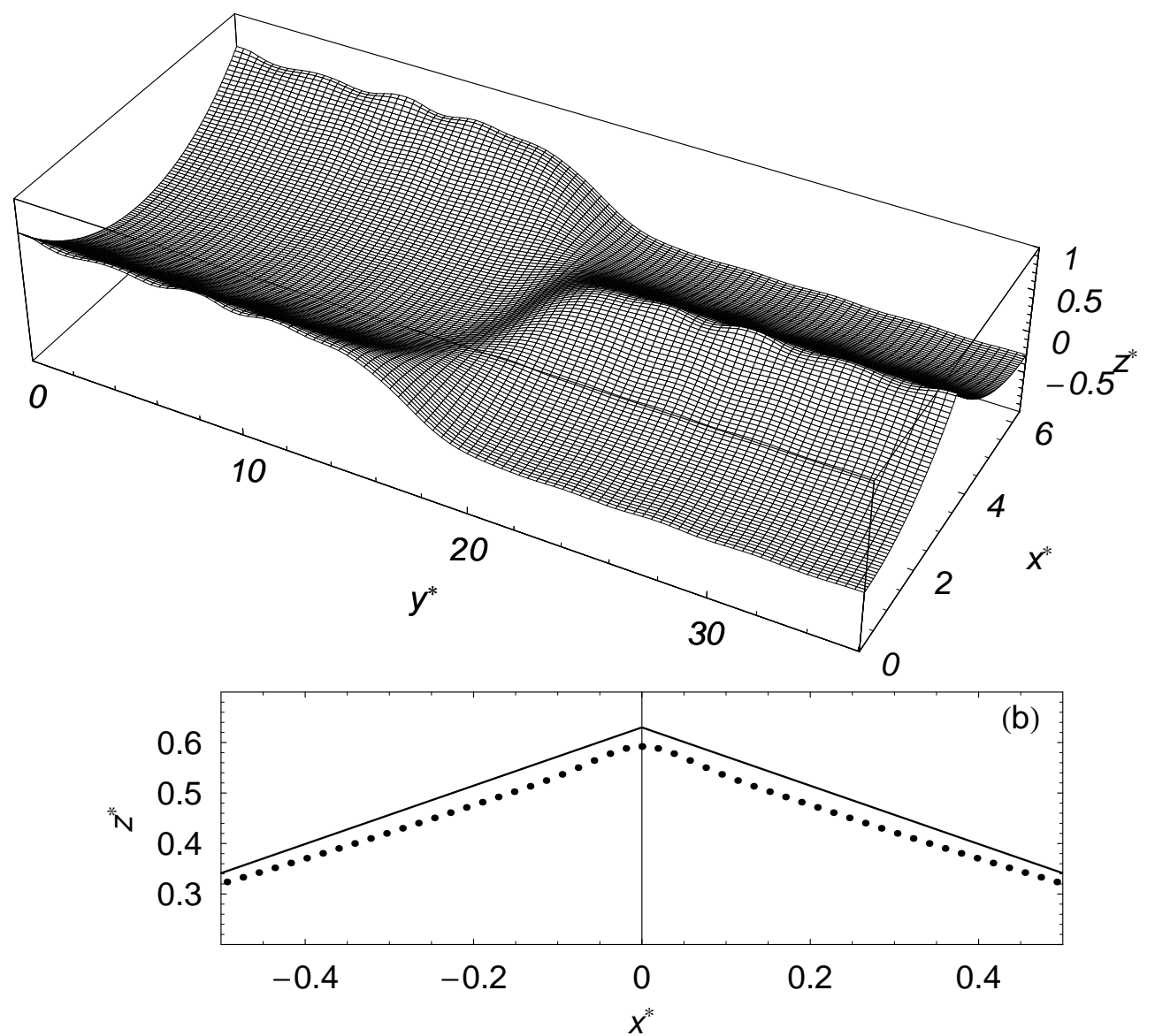

Figure 16. (a) Almost-limiting wave profile with $V_{c}=0.9903$ for $\theta=85^{\circ}$. (b) Cross-sectional view, $y^{*}=0$, of the wave profile near the peak: $\cdots$, the numerical result; - , the comparative profile $H^{*}\left(x^{*}, 0,0\right)=0.63-\left|x^{*}\right| / \sqrt{3}$ with an enclosed crest angle of $120^{\circ}$.

as

$$
\Phi \propto \Re\left[\left(x^{*}+\mathrm{i} z^{*}\right)^{3 / 2}\right]
$$

near the crest (e.g. $x^{*}=z^{*}=0$ ) and thus the crest is a singular point similar to that in a two-dimensional limiting progressive wave (Stokes (1847)). When $0^{\circ} \leqslant \theta \leqslant 47.5^{\circ}$, we can obtain an almost-limiting wave profile with a sharp corner because the velocity potential $\Phi$ at $x^{*}=x_{0}$ is expressed as

$$
\Phi \propto \bar{z}^{2}-\bar{y}^{2}
$$

near the crest (e.g. $\bar{y}=\bar{z}=0$, where $(\bar{y}, \bar{z})$ is obtained from $(y, z)$ by the rotation transformation to ensure that the surface fluid acceleration is downward). Thus, the crest is not a singular point but a saddle point similar to that in the limiting standing wave (Penney \& Price (1952); Okamura (1998)). We cannot prove that the crest is a saddle point, but have indirect numerical evidence that the limiting wave profile with a sharp corner is obtained by taking Taylor series expansion about the crest. Therefore, 


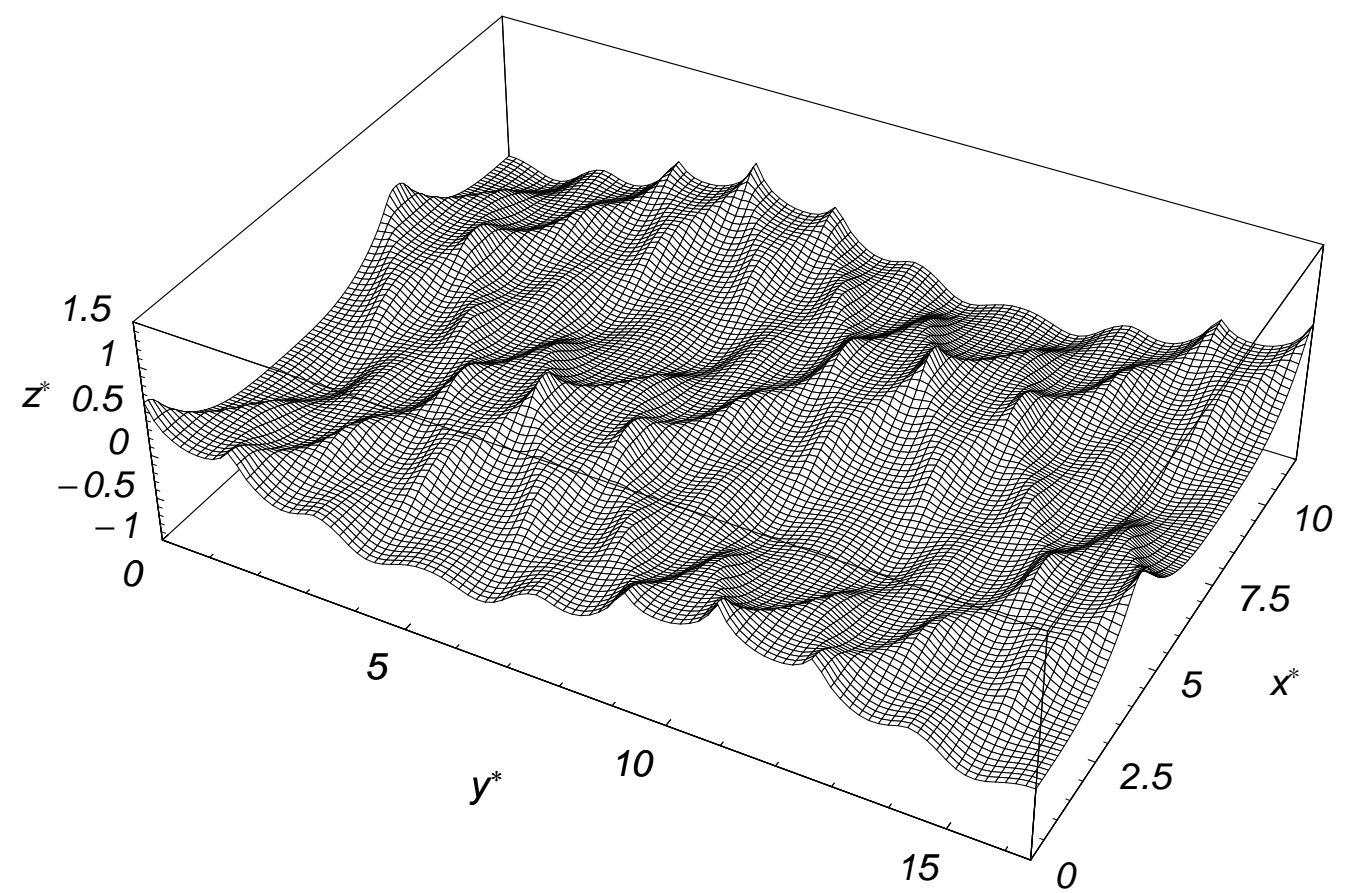

FiguRE 17. Almost-limiting wave profile with $V_{c}=0.9871$ for $\theta=55^{\circ}$, corresponding to the point $\left(A_{11}, A_{62}\right)=(0.297,0.0533)$ in the bifurcation diagram shown in figure $7(\mathrm{c})$.

the present method is a powerful method for investigating the characteristics of limiting short-crested waves, except for the sharp corner in limiting waves with $V_{c} \approx 1$.

I would like to acknowledge useful discussion with Dr. M. Ioualalen. I also thank one of the referees to inform me of the exact relation $(2.12)$.

\section{Appendix A. Calculation of the Jacobian matrix}

Here, we show how to calculate the elements of the Jacobian matrix, $\partial F_{l m} / \partial A_{k j}$, $\partial F_{l m} / \partial G, \partial W / \partial A_{k j}, \partial W / \partial G$.

$$
\frac{\partial F_{l m}}{\partial A_{k j}}=\int_{0}^{\pi} d T \int_{0}^{\pi} d Y\left(\frac{\partial Q}{\partial A_{k j}}+\frac{\partial Q}{\partial Z} \frac{\partial H}{\partial A_{k j}}\right) \cos (l Y) \sin (m T) .
$$

Since we have no information about $\partial H / \partial A_{k j}$ in the present formulation, we must eliminate it by using the relation,

$$
\frac{\partial P}{\partial A_{k j}}+\frac{\partial P}{\partial Z} \frac{\partial H}{\partial A_{k j}}=0
$$

which is obtained from (2.3). Elimination of $\partial f / \partial A_{k j}$ from (A 1) and (A 2) gives

$$
\frac{\partial F_{l m}}{\partial A_{k j}}=\int_{0}^{\pi} d T \int_{0}^{\pi} d Y\left(\frac{\partial Q}{\partial A_{k j}}-\frac{\partial Q}{\partial Z} \frac{\partial P / \partial A_{k j}}{\partial P / \partial Z}\right) \cos (l Y) \sin (m T) .
$$


Similarly, we obtain the other components of the Jacobian matrix:

$$
\begin{gathered}
\frac{\partial F_{l m}}{\partial G}=\int_{0}^{\pi} d T \int_{0}^{\pi} d Y\left(\frac{\partial Q}{\partial G}-\frac{\partial Q}{\partial Z} \frac{\partial P / \partial G}{\partial P / \partial Z}\right) \cos (l Y) \sin (m T), \\
\frac{\partial W}{\partial A_{k j}}=\left.\frac{\partial P / \partial A_{k j}}{\partial P / \partial Z}\right|_{Y=T=0}-\left.\frac{\partial P / \partial A_{k j}}{\partial P / \partial Z}\right|_{Y=\pi, T=0}, \\
\frac{\partial W}{\partial G}=\left.\frac{\partial P / \partial G}{\partial P / \partial Z}\right|_{Y=T=0}-\left.\frac{\partial P / \partial G}{\partial P / \partial Z}\right|_{Y=\pi, T=0} .
\end{gathered}
$$

Practical calculation of the Jacobian matrix requires the following relations:

$$
\begin{aligned}
& \frac{\partial P}{\partial Z}=-\Phi_{Z T}+p^{2} \Phi_{T} \Phi_{Z T}+q^{2} \Phi_{Y} \Phi_{Y Z}+\Phi_{Z} \Phi_{Z Z}+G \\
& \frac{\partial P}{\partial A_{k j}}=\left[j\left(p^{2} \Phi_{T}-1\right) \cos (k Y) \cos (j T)-q^{2} k \Phi_{Y} \sin (k Y) \sin (j T)\right. \\
& \left.+\alpha_{k j} \Phi_{Z} \cos (k Y) \sin (j T)\right] e^{\alpha_{k j} Z}, \\
& \frac{\partial P}{\partial G}=Z \\
& \frac{\partial Q}{\partial Z}=\Phi_{Z T T}+p^{2} \Phi_{Z T}\left(-2 \Phi_{T T}+p^{2} \Phi_{T} \Phi_{T T}+q^{2} \Phi_{Y} \Phi_{Y T}+\Phi_{Z} \Phi_{Z T}\right)+p^{2} \Phi_{T}\left(-2 \Phi_{Z T T}\right. \\
& \left.+p^{2} \Phi_{Z T} \Phi_{T T}+p^{2} \Phi_{T} \Phi_{Z T T}+q^{2} \Phi_{Y Z} \Phi_{Y T}+q^{2} \Phi_{Y} \Phi_{Y Z T}+\Phi_{Z Z} \Phi_{Z T}+\Phi_{Z} \Phi_{Z Z T}\right) \\
& +q^{2} \Phi_{Y Z}\left(-2 \Phi_{Y T}+p^{2} \Phi_{T} \Phi_{Y T}+q^{2} \Phi_{Y} \Phi_{Y Y}+\Phi_{Z} \Phi_{Y Z}\right)+q^{2} \Phi_{Y}\left(-2 \Phi_{Y Z T}\right. \\
& \left.+p^{2} \Phi_{Z T} \Phi_{Y T}+p^{2} \Phi_{T} \Phi_{Y Z T}+q^{2} \Phi_{Y Z} \Phi_{Y Y}+q^{2} \Phi_{Y} \Phi_{Y Y Z}+\Phi_{Z Z} \Phi_{Y Z}+\Phi_{Z} \Phi_{Y Z Z}\right) \\
& +\Phi_{Z Z}\left(-2 \Phi_{Z T}+p^{2} \Phi_{T} \Phi_{Z T}+q^{2} \Phi_{Y} \Phi_{Y Z}+\Phi_{Z} \Phi_{Z Z}+G\right)+\Phi_{Z}\left(-2 \Phi_{Z Z T}\right. \\
& \left.+p^{2} \Phi_{Z T}^{2}+p^{2} \Phi_{T} \Phi_{Z Z T}+q^{2} \Phi_{Y Z}^{2}+q^{2} \Phi_{Y} \Phi_{Y Z Z}+\Phi_{Z Z}^{2}+\Phi_{Z} \Phi_{Z Z Z}\right), \\
& \frac{\partial Q}{\partial A_{k j}}=\left[2 j\left\{p^{2}\left(-\Phi_{T T}+p^{2} \Phi_{T} \Phi_{T T}+q^{2} \Phi_{Y} \Phi_{Y T}+\Phi_{Z} \Phi_{Z T}+\alpha_{k j} \Phi_{T} \Phi_{Z}\right)-\alpha_{k j} \Phi_{Z}\right\}\right. \\
& \times \cos (k Y) \cos (j T)+\left\{-j^{2}+\alpha_{k j}\left(\alpha_{k j} \Phi_{Z}-2 \Phi_{Z T}+p^{2} \Phi_{T} \Phi_{Z T}+2 \Phi_{Z} \Phi_{Z Z}+G\right)\right. \\
& \left.+p^{2} \Phi_{T}\left(2 j^{2}-p^{2} j^{2} \Phi_{T}+\alpha_{k j} \Phi_{Z T}\right)+q^{2} \Phi_{Y}\left(2 \alpha_{k j} \Phi_{Y Z}-q^{2} k^{2} \Phi_{Y}\right)\right\} \cos (k Y) \sin (j T) \\
& -2 k q^{2}\left(-\Phi_{Y T}+p^{2} \Phi_{T} \Phi_{Y T}+q^{2} \Phi_{Y} \Phi_{Y Y}+\Phi_{Z} \Phi_{Y Z}+\alpha_{k j} \Phi_{Y} \Phi_{Z}\right) \sin (k Y) \sin (j T) \\
& \left.+2 q^{2} k j \Phi_{Y}\left(1-p^{2} \Phi_{T}\right) \sin (k Y) \cos (j T)\right] e^{\alpha_{k j} Z}, \\
& \frac{\partial Q}{\partial G}=\Phi_{Z}
\end{aligned}
$$

\section{REFERENCES}

Akylas, T. R. 1994 Three-dimensional long water-wave phenomena. Annu. Rev. Fluid Mech. 26, 191-210.

BRIDGES, T. J. 2009 Wave breaking and the surface velocity field for three-dimensional water waves. Nonlinearity 22, 947-953.

DiAs, F. \& BRIDGEs, T. J. 2006 The numerical computation of freely propagating timedependent irrotational water waves. Fluid Dyn. Res. 38, 803-830.

DiAs, F. \& Kharif, C. 1999 Nonlinear gravity and capillary-gravity waves. Annu. Rev. Fluid Mech. 31, 301-346. 
Hsu, J. R. C., Tsuchiya, Y. \& Silvester, R. 1979 Third-order approximation to short-crested waves. J. Fluid Mech. 90, 179-196.

IOUALALEn, M. \& KHARIF, C. 1993 Stability of three-dimensional progressive gravity-waves on deep-water to superharmonic disturbances. Eur. J. Mech. B/Fluids 12, 401-414.

Ioualalen, M. \& Okamura, M. 2002 Structure of the instability associated with harmonic resonance of short-crested waves. J. Phys. Oceanogr. 32, 1331-1337.

Ioualalen, M., Okamura, M., Cornier, S., Kharif, C. \& Roberts, A. J. 2006 Computation of short-crested deepwater waves. J. Waterw. Port C.-ASCE 132, 157-165.

Kimmoun, O., Branger, H. \& Kharif, C. 1999 On short-crested waves: experimental and analytical investigations. Eur. J. Mech. B/Fluids 18, 889-930.

Martin, D. U. \& Yuen, H. C. 1980 Quasi-recurring energy leakage in the two-spacedimensional nonlinear Schrödinger equation. Phys. Fluids 23, 881-883.

Mercer, G. N. \& Roberts, A. J. 1992 Standing waves in deep-water: Their stability and extreme form. Phys. Fluids A 4, 259-269.

Окамото, H. \& Shou, M. 2001 The mathematical theory of permanent progressive waterwaves. Singapore: World Scientific.

Okamura, M. 1996 Notes on short-crested waves in deep water. J. Phys. Soc. Jpn. 65, 28412845.

OKAmuRA, M. 1998 On the enclosed crest angle of the limiting profile of standing waves. Wave Motion 28, 79-87.

Okamura, M. 2003 Standing gravity waves of large amplitude in deep water. Wave Motion 37, $173-182$.

Penney, W. G. \& Price, A. T. 1952 Finite periodic stationary gravity waves in a perfect liquid. part II. Phil. Trans. R. Soc. London A 244, 254-284.

Perlin, M. \& Schultz, W. W. 2000 Capillary effects on surface waves. Annu. Rev. Fluid Mech. 32, 241-274.

Roberts, A. J. 1983 Highly non-linear short-crested water-waves. J. Fluid Mech. 135, 301-321.

Roberts, A. J. \& Peregrine, D. H. 1983 Notes on long-crested water-waves. J. Fluid Mech. 135, 323-335.

Stokes, G. G. 1847 On the theory of oscillatory waves. Trans. Cambridge Phil. Soc. 8, 441-455.

TANAKA, M. 1993 Mach reflection of a large-amplitude solitary wave. J. Fluid Mech. 248, 637661.

Tsai, C. P. \& Jeng, D. S. 1994 Numerical fourier solutions of standing waves in finite water depth. Appl. Ocean Res. 16, 185-193.

Williams, J. M. 1981 Limiting gravity-waves in water of finite depth. Phil. Trans. R. Soc. London A 302, 139-188.

YAMADA, H. 1957 Highest waves of permanent type on the surface of deep water. Rep. Res. Inst. Appl. Mech. Kyushu Univ. 5, 37-52. 